\title{
Steady state, oscillations and chaotic behavior of a gas inside a cylinder with a mobile piston controlled by PI and nonlinear control
}

\author{
Manuel Pérez-Molina ; Javier Gil Chica ${ }^{\mathrm{b}}$, Elena Fernández Varóc, \\ Manuel F. Pérez-Polo ${ }^{\mathrm{d}^{*}}$,
}

a b d Departamento de Física, Ingeniería de Sistemas y Teoría de la Señal, Escuela Politécnica Superior, Universidad de Alicante. ${ }^{c}$ Departamento de Óptica, Campus de San Vicente, Alicante, Spain. Fax 965903682. ${ }^{\mathrm{a} E}$-mail: ma perez_m@,hotmail.com. ${ }^{b} \mathrm{E}-$ mail: gil@dfists.ua.es. ${ }^{c} E-m a i l:$ elena.fernandez@ua.es ${ }^{d}$ E-mail: manolo@dfists.ua.es. Phone: +34965909673

\begin{abstract}
This paper analyzes the behavior of nitrogen inside a closed cylinder with a mobile piston actuated by a nonlinear spring, a viscous damper and a control force which compensates partially the effect of the high gas pressure. Two helical heating coils are placed inside the cylinder and with their flow rates controlled by means of a linear controller of type proportional plus integral (PI) and another nonlinear control law to provide an approximately isothermal gas behavior. Based on the analysis of the mechanical and thermal subsystems and the control laws, a justification of the parameter values is presented and corroborated through analytical solutions that are obtained by approximate methods. To investigate the thermodynamic equilibrium conditions, the Soave-Redlich-Kwong and the Redlich-Kwong state equations are analyzed and compared, showing that the Soave-Redlich-Kwong equation is superior. The Melnikov method has been used to obtain sufficient conditions for chaotic behavior, which has also been investigated by means of the sensitive dependence, Lyapunov exponents and the power spectral density. The validity of the proposed model has been analyzed by using the compressibility chart for the nitrogen, and the analytical calculations have been verified through full numerical simulations.
\end{abstract}

Keywords: Real gas state equations, steady-state behavior, oscillating and chaotic behavior, linear and nonlinear control. 


\section{Introduction}

The study of classical thermodynamics is generally based on the assumption that the system is at an equilibrium state and passes to another one by means of some transformation type. This idea has been applied in many problems related with all areas of science and engineering, for which there exists a vast literature. Some significant examples are indicated in Refs [1-5].

Many thermodynamic problems are related to the exchange of heat and work between a fluid and a mechanical system [4-7], as it occurs with the explosion engines. In these interactions between mechanical systems and fluids, the motion of the mobile parts is generally assumed to be regular. However, the interaction between a mechanical system with chaotic motions of their mobile parts and a gas at high pressure that is regarded as non-ideal has been less studied. In this case, the difficulty arises from the analysis of the chaotic motion and its interaction with the fluid as well as the need of an accurate state equation for the non-ideal gas [8-14].

On the other hand, the use of one o more control systems for positioning the mobile parts of a mechanical system as well as to lead the state variables of the gas (such as pressure, volume or temperature) to a desired set point is an interesting problem from a practical viewpoint, even considering simple laws of linear control such as the proportional plus integral (PI) controller [15-17]. More complicated control laws such as the ones based on differential geometry are less frequent in the control of mechanical and thermal subsystems. Some examples of such problems can be found in Refs [18-20]. Other control strategies, such as partial control are potentially interesting to be applied in these studies [21].

The analysis of chaotic behavior in the interaction between mechanical systems with mobile parts and thermal systems can be interesting in statistic mechanics and in problems of phase transitions [1-3], although this topic has not been widely studied. This is probably due to the inherent difficulty to find out when an oscillation can be regarded as chaotic. In this sense, the classic Melnikov's method only provides necessary conditions for chaos, and the required mathematical calculations are 
cumbersome in many cases. Other evidences of chaos such as the Lyapunov exponents may also be difficult to obtain [22-27].

In this work we analyze the behavior of nitrogen regarding it as a non-ideal gas, since the work pressure is above the critical one. This non-ideal gas is inside a cylinder that is considered to be adiabatic with a mobile piston in the horizontal direction. The piston motion is controlled by an external force, by a nonlinear spring and by a viscous damper. The external force which acts over the piston has the purpose of providing the necessary balance between all the forces to maintain the piston motion within the cylinder limits as well as providing oscillatory and chaotic motions.

Two additional control systems are applied to two heating helical coils which are inside the cylinder with the purpose of maintaining the gas temperature at a predetermined set point and supplying the necessary heat to assure the stability of the thermal subsystem. With an adequate choice of the parameter values, it is shown that the piston can reach several equilibrium points as well as chaotic motions that are transmitted to the gas. On the other hand, it is shown that this control system allows decoupling between the mechanical and thermal subsystems.

The analysis of the thermal subsystem is carried out by using the Soave-RedlichKwong (SRK) and the Redlich-Kwong (RK) state equations, which have three and two adjustable parameters respectively. Other empirical state equations with more than three adjustable parameters can be found in Refs [5], [28]. In addition, the variation of the specific heat with respect to the pressure and the fulfillment of the thermodynamic equilibrium condition have been analyzed.

Two approximate methods (based on the Laplace transform and the linearization of the system) are used to verify the accurateness of the simulation results. Besides, chaotic motion has been verified by means of sensitive dependence, Lyapunov exponents and power spectral density. Finally, a discussion regarding the applicability of the proposed model is presented taking into account the compressibility diagram of the nitrogen and the stability of the control system. 
The system presented in this paper can be used to estimate the thermodynamic properties of a gas or a mixture of real gases and to compare them with experimental data. In addition, the design of different control strategies to achieve a prescribed dynamical behavior can also be investigated. A remarkable example could be the estimation of the first and second coefficients of Virial state equation. For this purpose, a control system is necessary to drive the gas pressure to a value as low as possible, and thus the first and second derivative of the compressibility coefficient with respect to the (low) pressure could be used to compare the Virial state equation with the SoaveRedlich-Kwong and Redlich-Kwong state equations analyzed in this paper [1], [3], [5], [28], [29], [30].

\section{Description of the system and mathematical model}

The analyzed system is formed by a closed cylinder to which a mobile piston is anchored by means of a nonlinear spring and a damper. Inside the cylinder there is nitrogen, which will be regarded as a real gas since the working pressure of the system is above the critical pressure (which is 33.94 atmospheres). The scheme of the device with two control loops is shown in figure 1 a: the control loop 1 is linear whereas the control loop 2 is nonlinear. The bumpers $B$ provide a non-accessible space for the piston, where there are two heater helical coils $C$ and $C_{l}$ whose objective is to heat the gas. It is assumed that the net force of the nonlinear spring at the distance $d_{1}$ is zero, whereas the damper is considered to be linear with a damping coefficient denoted by $b$. The atmospheric pressure is denoted by $P_{a}$, and an external control force $F^{\prime}(t)$ is applied to ensure a motion of the piston within the cylinder limits. Since the device is formed by a mechanical and a thermal subsystem, the model equations are deduced separately from each subsystem as follows:

Figure 1

a) Equation of the mobile piston.

The control total force on the mobile piston is given by: 


$$
F(t)=F^{\prime}(t)+S \cdot P_{a}
$$

where $S$ is the cross sectional area of the piston. In accordance with the reference system shown in Fig 1 it is deduced that the gas volume $V(t)$ and the mobile piston displacement $x(t)$ can be written as a function of an effective volume $V_{e}$ as:

$$
\left.\begin{array}{c}
x_{1}(t)=L+L_{1}-c / 2-x(t)=d-x(t) ; d=L+L_{1}-c / 2 \\
V(t)=S \cdot x_{1}(t)-V_{c}-V_{s d}=V_{e}-S \cdot x(t) \Rightarrow x(t)=\frac{V_{e}-V(t)}{S} \\
V_{e}=S \cdot d-V_{c}-V_{s d}
\end{array}\right\}
$$

where $V_{c}$ and $V_{s d}$ are the volumes of the heater coils and the spring-damper respectively. The nonlinear spring and the damper forces can be defined as:

$$
\left.\begin{array}{c}
F_{s}(t)=K_{1}\left[x_{1}(t)-d_{1}\right]+K_{2}\left[x_{1}(t)-d_{1}\right]^{3} \\
F_{d}(t)=b \frac{d\left[x_{1}(t)-d_{1}\right]}{d t}
\end{array}\right\}
$$

According to Eqs (2) and (3), the motion equation of the mobile piston can be written as follows:

$m \frac{d^{2} x(t)}{d t^{2}}=F(t)-S \cdot P(t)+K_{1}\left[d-d_{1}-x(t)\right]+K_{2}\left[d-d_{1}-x(t)\right]^{3}+b \frac{d\left[d-d_{1}-x(t)\right]}{d t}$

where $m$ is the mass of the mobile piston and $P(t)$ is the gas pressure. Introducing the nomenclature $\bar{d}_{1}=d_{1}-\left(V_{c}+V_{s d}\right) / S$, Eqs (2) and (3) allow to write the motion equation of the piston as a function of the gas volume $V(t)$ to yield:

$$
\frac{d^{2} V(t)}{d t^{2}}=\frac{S^{2}}{m} P(t)-\frac{S}{m} F(t)-\frac{K_{1}}{m}\left[V(t)-S \cdot \bar{d}_{1}\right]-\frac{K_{2}}{m S^{2}}\left[V(t)-S \cdot \bar{d}_{1}\right]^{3}-\frac{b}{m} \frac{d V(t)}{d t}
$$

b) Energy balance in the thermal subsystem. 
Assuming that the work done on the gas is reversible and of mechanical type, the third Maxwell relation [1-5] allows to obtain the most general expression for the first principle of Thermodynamics, which can be expressed as:

$$
n c_{v}(t) \frac{d T(t)}{d t}+\left[T\left(\frac{\partial P}{\partial T}\right)_{v}-P\right] \frac{d V(t)}{d t}=Q_{r e v}(t)-P \frac{d V(t)}{d t}
$$

where $n$ is the number of moles of the gas and $c_{v}(t)$ is the specific heat of the gas at constant volume, which depends on the temperature and the pressure and thus it also depends on the time (since both the temperature and the pressure are time functions). The calculation of $c_{v}(t)$ will be analyzed in section 4 . The variable $V$ is the gas volume and $v=V / n$ is the molar volume, whereas $P$ and $T$ are the gas pressure and temperature respectively and $Q_{\text {rev }}$ is the reversible heat flux (Joules/s) supplied by the heating coils. Hereafter, an expression of the form $(\partial x / \partial y)_{z}$ will denote partial derivation of $x$ respect to $y$ at a constant $z$, taking $x, y$ and $z$ as any of the state variables $v, P, T$ of the gas.

To model the heating coils, the following assumptions regarding the heat transmission are considered:

- The velocity of the heating fluid in the coils is sufficiently high so that the variation of temperature in the coil can be regarded as negligible.

- The fluid volume inside the coils is small, and thus its temperature remains almost constant.

- The heat flux generated in the heating coil C (Fig 1) is given by:

$$
Q(t)=K_{s} F_{v}(t)
$$

where $F_{v}(t)$ is the flow rate of the heating fluid and $K_{s}$ is a constant which depends on the material and the layout of the heating coils.

- Denoting by $h$ the overall heat transmission coefficient [6-7] and denoting by $A_{t}$ the heat transmission area we can write that $h A_{t}\left[T_{c}-T(t)\right]=K_{s} F_{v}(t)$, where $T_{c}$ is the temperature of the fluid inside the coils and $T(t)$ is the gas temperature. 
- Assuming that the heating coils have an external diameter $D_{c}$ and an internal diameter $d_{c}$ with $N$ turns, the constant of the heaters $K_{s}$ can be estimated as follows:

$$
K_{s}=\frac{4\left(1+3.5 d_{c} / D_{c}\right) \pi D_{c} h N\left(T_{c}-T_{s}\right)}{d_{c} v_{f}}\left(\frac{k J}{m^{3}}\right)
$$

where $T_{S}$ is the set temperature of the gas and it is assumed that the control loop 1 (see Fig. 1) maintains the gas temperature very close to the set temperature, i.e. $T(t) \approx T_{s}$.

The parameter values of the whole system are shown in table 1, and the justification of their choice will be discussed in the next section.

Table 1

Next we are going to define the temperature control loop 1, which is a PI controller [15-17] defined by the following equations:

$$
\left.\begin{array}{c}
F_{v}(t)=K_{p}\left[T_{s}-T(t)+\frac{1}{\tau_{i}} \int_{0}^{t}\left[T_{s}-T(\tau)\right] d \tau\right] \\
\frac{d Q(t)}{d t}=-K_{p} K_{s} \frac{d T(t)}{d t}+\frac{K_{p} K_{s}}{\tau_{i}}\left[T_{s}-T(t)\right]
\end{array}\right\}
$$

where $\mathrm{K}_{\mathrm{p}}$ is the proportional constant and $\tau_{\mathrm{i}}$ is the reset time of the PI controller. The nonlinear control loop 2 can be defined in accordance with the layout shown in Fig 1 through the following equations:

$$
\left.\begin{array}{l}
F_{v 1}(t)=f(T)\left[\alpha K_{s} F_{v}(t)+\beta\left[T_{s}-T(t)\right]\right] \\
Q_{1}(t)=K_{s} f(T)\left[\alpha K_{s} F_{v}(t)+\beta\left[T_{s}-T(t)\right]\right]
\end{array}\right\}
$$


The previous control laws can be justified as follows. The heat flux in the heater coil $2(\mathrm{Eq}(10))$ is proportional to the flow rate of heating fluid in the heater coil 1 plus a control signal proportional to the temperature error signal. Consequently, this control law would become linear if $f(T)=1$. However, taking into account that the thermal subsystem is nonlinear, the introduction of an adequate function $f(T)$ may stabilize such subsystem. Therefore, the purpose of the control loop 1 is to stabilize the gas temperature at a desired set point temperature $T_{s}$, whereas the purpose of the control loop 2 is to stabilize the heat transmission to the gas by using the nonlinear function $f(T)$. Substituting Eq (10) into Eq (6) it is deduced that:

$$
\frac{d T(t)}{d t}=\frac{Q(t)}{n c_{v}(t)}+\frac{K_{s} f(T)}{n c_{v}(t)}\left[\alpha Q(t)+\beta\left[T_{s}-T(t)\right]\right]-\frac{T(t)}{n c_{v}(t)}\left(\frac{\partial P}{\partial T}\right)_{v} \frac{d V(t)}{d t}
$$

Taking $v(t)=V(t) / n, T(t)$ and $Q(t)$ as state variables, Eqs (5), (9) and (11) provide the mathematical model of the system. In addition, it is necessary to consider the state equation of the gas, for which we will analyze two state equations that are frequently used in the analysis of chemical engineering processes [5-28]: i) The Soave-RedlichKwong (SRK) equation [9-13] and ii) the Redlich-Kwong (RK) equation [8], [14], which are defined as follows:

i) Soave-Redlich-Kwong (SRK) equation:

$$
\begin{gathered}
P=\frac{R T}{v-b}-\frac{\bar{\alpha} a}{v(v+b)} ; \bar{\alpha}=\left[1+m_{i}\left(1-T_{r}^{0.5}\right)\right]^{2} ; m_{i}=0.480+1.574 \omega-0.176 \omega^{2} \\
a=0.42748 \frac{R^{2} T_{c}^{2}}{P_{c}} ; b=0.08664 \frac{R T_{c}}{P_{c}} ; T_{r}=\frac{T}{T_{c}} ; R=0.082 \frac{\mathrm{atm} \cdot \mathrm{liter}}{\mathrm{K} \cdot \mathrm{mol}}
\end{gathered}
$$

where $P, T$ and $v$ are the pressure, temperature and molar volume respectively, $R$ is the constant of gases, $P_{c}$ and $T_{c}$ are the critical pressure and temperature respectively, $T_{r}$ is the reduced temperature and $\omega$ is a parameter called Pitzer's acentric factor, which takes into account the symmetry properties of the gas molecule $[28,30]$.

ii) Redlich-Kwong (RK) equation: 


$$
\begin{gathered}
P=\frac{R T}{v-b}-\frac{a}{v(v+b) T^{1 / 2}} ; R=0.082 \frac{\mathrm{atm} \cdot \text { liter }}{\mathrm{K} \cdot \mathrm{mol}} \\
a=0.4275 \frac{R^{2} T_{c}^{5 / 2}}{P_{c}} ; b=0.08664 \frac{R T_{c}}{P_{c}}
\end{gathered}
$$

where the notation is the same as in Eq (12). It should be noted that the use of Eqs (12) and (13) to calculate $(\partial P / \partial T)_{v}$ in Eq (11) implies expressing $P$ in atmospheres and $v$ in liters, so if $c_{v}$ is expressed in the usual units of joules $/(\mathrm{mol} \cdot \mathrm{K})$ then the term $(\partial P / \partial T)_{v}$ must be multiplied by $1.0325 \cdot 10^{2}$ (i.e. $1.0325 \cdot 10^{5} \mathrm{~N} / \mathrm{m}^{2} / \mathrm{atm} \cdot 10^{-3} \mathrm{~m}^{3} /$ liters). As it will be shown later, the SRK equation provides more reliable results that the RK equation.

In order to simplify the stability analysis of Eqs (8) and (10), the nonlinear function $f(T)$ of $\mathrm{Eq}(10)$ is defined as follows:

$$
f(T)=c_{v}(t)\left[1-\frac{Q_{s}}{Q(t)}\right]-\frac{Q_{s}}{\alpha K_{s} Q(T)}
$$

where $Q_{s}$ is the steady state value of $Q(t)$ and $T_{s}$ is the set point temperature. Substituting Eq (14) into Eq (11) it is deduced that:

$$
\begin{aligned}
& \frac{d T(t)}{d t}=\frac{\alpha K_{s}}{n}\left[Q(t)-Q_{s}\right]-\frac{\beta K_{s}}{n}\left[T(t)-T_{s}\right]+\frac{Q(t)-Q_{s}}{n c_{v}(t)}+ \\
& +\frac{\beta}{n}\left[\frac{T(t)-T_{s}}{Q(T)}\right]\left[K_{s}+\frac{1}{\alpha c_{v}(t)}\right]-\frac{T(t)}{n c_{v}(t)}\left(\frac{\partial P}{\partial T}\right)_{v} \frac{d V(t)}{d t}
\end{aligned}
$$

To simplify the analysis of the system it is convenient to introduce the following deviation variables:

$$
\left.\left.\begin{array}{c}
T^{\prime}(t)=T(t)-T_{s} \\
Q^{\prime}(t)=Q(t)-Q_{s}
\end{array}\right\} \Rightarrow \begin{array}{c}
T(t)=T^{\prime}(t)+T_{s} \\
Q(t)=Q^{\prime}(t)+Q_{s}
\end{array}\right\}
$$

Eqs (9) and (15) can be written as follows: 


$$
\begin{gathered}
\frac{d T^{\prime}(t)}{d t}=-\frac{\beta K_{s}}{n} T^{\prime}(t)+\frac{\alpha K_{s}}{n} Q^{\prime}(t)+\frac{Q^{\prime}(t)}{n c_{v}(t)}+\left[K_{s}+\frac{1}{\alpha c_{v}(t)}\right] \frac{\beta Q_{s}}{n} \frac{T^{\prime}(t)}{\left[Q^{\prime}(t)+Q_{s}\right]} \\
-\left[\frac{T^{\prime}(t)+T_{s}}{n c_{v}(t)}\right]\left(\frac{\partial P}{\partial T}\right)_{v} \frac{d V(t)}{d t} \\
\frac{d Q^{\prime}(t)}{d t}=-K_{p} K_{s} \frac{d T^{\prime}(t)}{d t}-\frac{K_{p} K_{s}}{\tau_{i}} T^{\prime}(t)
\end{gathered}
$$

On the other hand, for the sake of simplifying the treatment of the mechanical subsystem defined by Eq (5), we shall introduce the following variables:

$$
\left.\begin{array}{c}
V^{\prime}(t)=V(t)-V_{s} ; V_{s e}=V_{s}-S \cdot \bar{d}_{1} \\
P^{\prime}(t)=P(t)-P_{s} ; F^{\prime}(t)=F(t)-F_{s}
\end{array}\right\}
$$

where $V_{s}$ is the accessible piston volume, $P_{s}$ is an equilibrium pressure and $F_{S}$ is a equilibrium force. Taking into account the equilibrium equations (16), Eqs (5), (18) and (19) allow to express the system equations as:

$$
\left.\begin{array}{c}
\frac{d^{2} V^{\prime}(t)}{d t^{2}}=-\frac{K_{1}}{m}\left[V^{\prime}(t)+V_{s e}\right]-\frac{K_{2}}{m S^{2}}\left[V^{\prime}(t)+V_{s e}\right]^{3}-\frac{b}{m} \frac{d V^{\prime}(t)}{d t}+ \\
+\frac{S^{2}}{m}\left[P^{\prime}(t)+P_{s}\right]-\frac{S}{m}\left[F^{\prime}(t)+F_{s}\right] ; \quad V_{s e}=V_{s}-S \cdot \bar{d}_{1} \\
\frac{d T^{\prime}(t)}{d t}=-\frac{\beta K_{s}}{n} T^{\prime}(t)+\frac{\alpha K_{s}}{n} Q^{\prime}(t)+\frac{Q^{\prime}(t)}{n c_{v}(t)}+ \\
+\left[K_{s}+\frac{1}{\left.\alpha c_{v}(t)\right]}\right] \frac{\beta Q_{s} T^{\prime}(t)}{n\left[Q^{\prime}(t)+Q_{s}\right]}-\left[\frac{T^{\prime}(t)+T_{s}}{\left.n c_{v}(t)\right]\left(\frac{\partial P}{\partial T}\right)_{v} \frac{d V^{\prime}(t)}{d t}}\right. \\
\frac{d Q^{\prime}(t)}{d t}=-\left(\frac{K_{s} K_{p}}{\tau_{i}}-\frac{\beta K_{s}^{2} K_{p}}{n}\right) T^{\prime}(t)-\frac{\alpha K_{s}^{2} K_{p}}{n} Q^{\prime}(t)-\frac{K_{s} K_{p} Q^{\prime}(t)}{n c_{v}(t)}- \\
-K_{s} K_{p}\left[K_{s}+\frac{1}{\alpha c_{v}(t)}\right] \frac{\beta Q_{s} T^{\prime}(t)}{n\left[Q^{\prime}(t)+Q_{s}\right]}+\left[\frac{K_{s} K_{p}\left[T^{\prime}(t)+T_{s}\right]}{n c_{v}(t)}\right]\left(\frac{\partial P}{\partial T}\right)_{v} \frac{d V^{\prime}(t)}{d t}
\end{array}\right\}
$$

It is important to remark that from Eqs (5) and (19) it follows that $V(t)-S \bar{d}_{1}$ is the part of the cylinder volume that is accessible for the piston motion (see Fig 1). Equations (20) together with the SRK or RK state equations (Eqs (12) or (13)) conform 
the mathematical model of the device. Next we are going to analyze admissible parameter values for the thermal and mechanical subsystems including the control systems. It should be noted that we are interested in the behavior of the system in steady state and in oscillating regime as well as in chaotic regime, since we intend to study the conditions under which the SRK and RK state equations can be applied in accordance with the equations of the classical equilibrium thermodynamics.

\section{Parameter values, stability of the equilibrium points and homoclinic orbits}

A careful and detailed analysis of the parameter values is necessary to warrant a physically valid model of the system. Therefore, in this section we are going to justify the parameter values shown in table 1 to analyze the stability of the equilibrium points of the system. In addition, we shall study the conditions under which homoclinic orbits and oscillating behaviors may appear. These issues will be important in the analysis of the chaotic behavior, as we will see later.

\subsection{Parameter values and equilibrium points of the thermal subsystem}

Substituting the term $d T^{\prime}(t) / d t$ of Eq (19) into Eq (18) we obtain a pair of nonlinear differential equations associated to the thermal subsystem. The linear part of these equations has the following eigenvalues:

$$
\lambda_{3,4}=-\frac{1}{2}\left(\frac{\beta K_{s}}{n}+\frac{\alpha K_{s}^{2} K_{p}}{n}\right) \pm \frac{1}{2} \sqrt{\left(\frac{\beta K_{s}}{n}+\frac{\alpha K_{s}^{2} K_{p}}{n}\right)^{2}-4 K_{s}^{2} K_{p} \frac{\alpha}{n \tau_{i}}}
$$

Now we shall consider the following inequality:

$$
4 K_{s}^{2} K_{p} \frac{\alpha}{n \tau_{i}}>\left(\frac{\beta K_{s}}{n}+\frac{\alpha K_{s}^{2} K_{p}}{n}\right)^{2} \Rightarrow \tau_{i}<\frac{4 n \alpha K_{p}}{\left(\beta+\alpha K_{s} K_{p}\right)^{2}}
$$

According to Eq (21), if the inequality of Eq (22) is fulfilled then the equilibrium point $\left(T_{s}, Q_{s}\right)$ will be a stable focus. On the other hand, introducing a variable factor $f_{c}$ 
such that $0<f_{c}<1$, the inequality (22) can be transformed into an equality from which it is possible to obtain the maximum value of the reset time $\tau_{\text {imax }}$ taking $\alpha$ as independent variable, i.e.:

$$
\tau_{i}=f_{c} \frac{4 n \alpha K_{p}}{\left(\beta+\alpha K_{s} K_{p}\right)^{2}} \Rightarrow \alpha_{m}=\frac{\beta}{K_{s} K_{p}} \Rightarrow \tau_{i \max }=f_{c} \frac{n}{\beta K_{s}}
$$

From Eqs (23) it is possible to analyze the values of the reset time as a function of the values of $\beta, K_{s}$ and $K_{p}$. Initially, the number of nitrogen moles can be approximated considering it as an ideal gas at a temperature of $T_{s}=200 \mathrm{~K}$ and a pressure of $40 \mathrm{~atm}$, with which we obtain 11.494 moles of nitrogen.

The value of $K_{p}$ can be obtained from the equality $K_{p}=F_{v s} /\left(T_{c}-T_{s}\right)$ taking tentatively $T_{c}-T_{s}=10 \mathrm{~K}$ and taking $F_{v s}=\pi d_{c}^{2} v_{f} / 4$, where $v_{f}$ is the fluid velocity in the heating coils. The product $K_{s} \cdot K_{p}=1.37050$ has been chosen in order to obtain reasonable values for the control signal. Now, from Eq (8), for a fluid velocity of $v_{f}=3$ $\mathrm{ft} / \mathrm{s}$ and from values for the global heat transmission within the range 560-1400 $B T U / h \cdot f t^{2 o} F$ [6-7], we obtain a mean value of $K_{s}=7.5020 \cdot 10^{8} \mathrm{~J} / \mathrm{m}^{3}$ and therefore $K_{p}=$ $1.8268 \cdot 10^{-9} \mathrm{~m}^{3} / \mathrm{s} \cdot \mathrm{K}$. Consequently, the exact number of nitrogen moles is 15.757 , which is deduced from Eqs (12) and (13) and from the gas volume indicated in table 1. Fig 2 has been plotted considering the previous values. The value $f_{c}=0.001$ has been chosen to avoid large values for the reset time and therefore to obtain suitable values for the integral action (which is proportional to $1 / \tau_{i}$ ). It should be noted that the value of the reset time coincides with the maximum between $K_{p}$ and $K_{s}$ in all the previously specified subplots, i.e. $\tau_{i}=\tau_{\text {imax }}=0.1576 \mathrm{~s}$. Consequently, the values of $\alpha$ and $\beta$ are given by $\alpha=9.7262 \cdot 10^{-11}\left(\mathrm{~m}^{3} \cdot \mathrm{mole} \cdot \mathrm{K} / \mathrm{J}^{2}\right)$ and $\beta=1.3330 \cdot 10^{-10}\left(\mathrm{~m}^{3} / \mathrm{J}\right)$, in accordance with Fig 2.

Figure 2

3.2 Parameter values and equilibrium points for the mechanical subsystem 
Taking into account that the pressure in the gas is very high, if such pressure were balanced only through the spring and damper then the values of $K_{1}, K_{2}$ and b would have to be very high, and thus the oscillation frequencies of the piston $\left(\sim K_{l} / m\right)$ would take prohibitive values. On the other hand, in order to study different behaviors of the gas it is necessary to ensure that the piston has several equilibrium points. With this purpose, we choose the force $F^{\prime}(t)$ in $\mathrm{Eq}(20)$ as follows:

$$
\frac{S}{m}\left[F^{\prime}(t)+F_{s}\right]=\frac{S^{2}}{m}\left[P^{\prime}(t)+P_{s}\right]-f_{p} \frac{b}{m} \frac{d V^{\prime}(t)}{d t}-\frac{K}{S^{2}}\left[V^{\prime}(t)+V_{s e}\right]^{2}-\frac{S^{2}}{m} P_{1 s}
$$

where $f_{p}$ is a variable factor such that the friction effect is considered when $f_{p}=0$ and removed when $f_{p}=1$. It is important to remark that the control law given by Eq (24) decouples the thermal and mechanical subsystems, since the mechanical system acts over the thermal one but its dynamics is independent from the thermal system because the effect of the gas pressure is cancelled through Eq (24). Substituting Eq (24) into the first equation (20) it is deduced that:

$$
\left.\begin{array}{c}
\frac{d^{2} V^{\prime}(t)}{d t^{2}}=-\frac{K_{1}}{m}\left[V^{\prime}(t)+V_{s e}\right]-\frac{K_{2}}{m S^{2}}\left[V^{\prime}(t)+V_{s e}\right]^{3}-\left(1-f_{p}\right) \frac{b}{m} \frac{d V^{\prime}(t)}{d t}+ \\
+\frac{K}{m S}\left[V^{\prime}(t)+V_{s e}\right]^{2}+\frac{S^{2}}{m} P_{1 s}
\end{array}\right\}
$$

On the other hand, denoting by $V_{p}(t)=V^{\prime}(t)+V_{s e}=V(t)-S \cdot \bar{d}_{1}$ the reachable volume of the piston when it is in an arbitrary position between the bumpers (B) and the end of the cylinder (see Fig 1), Eq (25) can be rewritten as follows:

$$
\frac{d^{2} V_{p}(t)}{d t^{2}}=-\frac{K_{1}}{m} V_{p}(t)-\frac{K_{2}}{m S^{2}} V_{p}^{3}(t)-\left(1-f_{p}\right) \frac{b}{m} \frac{d V_{p}(t)}{d t}+\frac{K}{m S} V_{p}^{3}(t)+\frac{S^{2}}{m} P_{1 s}
$$

Introducing the deviation variable $V_{p}^{\prime}(t)=V_{p}(t)-V_{s}$, Eq (26) can be rewritten in terms of the new variable $V_{p}^{\prime}(t)$. Consequently, in the equilibrium state with $V_{p}^{\prime}(t)=0$, the following equation is fulfilled: 


$$
y\left(V_{s}\right)=\frac{K_{2}}{S^{2}} V_{s}^{3}-\frac{K}{S} V_{s}^{2}+K_{1} V_{s}-S^{2} P_{1 s}=0
$$

We now aim to determine the values of $K_{1}, K_{2}, K$ and $P_{l s}$ for which we obtain three equilibrium points inside the accessible zone of piston movement. This means that Eq (27) must have a maximum and a minimum in this zone, and therefore the following condition must be verified:

$$
K^{2}>3 K_{1} K_{2} \Rightarrow K=\sqrt{3 f_{k} K_{1} K_{2}} ; f_{k}>1
$$

On the other hand, denoting by $d_{1 c}, d_{2 c}$ and $d_{3 c}$ the respective roots of Eq (27) within the region of the piston movement with $d_{1 c}>d_{1} ; d_{3 c}<L-c$ and $d_{1 c}<d_{2 c}<d_{3 c}$ (see table 1), and considering that $V_{s i}=S \cdot d_{i c}(i=1,2,3)$, the following relations can be found:

$$
\left[\begin{array}{lll}
-S^{2} d_{1 c}^{2} & S d_{1 c} & -1 \\
-S^{2} d_{2 c}^{2} & S d_{2 c} & -1 \\
-S^{2} d_{3 c}^{2} & S d_{3 c} & -1
\end{array}\right]\left[\begin{array}{l}
a_{2} \\
a_{1} \\
a_{0}
\end{array}\right]=\left[\begin{array}{l}
-S^{3} d_{1 c}^{3} \\
-S^{3} d_{2 c}^{3} \\
-S^{3} d_{3 c}^{3}
\end{array}\right] ; a_{2}=\frac{K S}{K_{2}} ; a_{1}=\frac{K_{1} S^{2}}{K_{2}} ; a_{0}=\frac{P_{1 s} S^{4}}{K_{2}}
$$

We are going to obtain the values of $K_{1}, K_{2}, K$ and $P_{1 s}$ for which three equilibrium points $d_{l c}, d_{2 c}$ and $d_{3 c}$ inside the zone of the piston movement are obtained. For this purpose, the following steps are carried out:

- We fix $d_{l c}$ and $d_{3 c}$, and consider a set of values for $d_{2 c}$ which is denoted by $d_{2 c v}$. Then Eq (29) is solved for each triplet $\left(d_{1 c}, d_{2 c}, d_{3 c}\right)$. Next we fix a set of values for $K_{l v}$ between 50 to $500 \mathrm{~N} / \mathrm{m}$ (taking into account that the piston mass is approximately $1 \mathrm{~kg}$ ). From the value of $a_{l}$ in Eq (29), the corresponding values of $K_{2 v}$ can be calculated.

- We choose a value $f_{k}>1$, and taking into account the previous values of $K_{l v}$ and $K_{2 v}$ and Eq (28) the corresponding values of $K_{v}$ are calculated.

- We choose a set of tentative values for $P_{1 s v}=K_{1 v} K_{v} / S K_{2 v}$. And finally, for a chosen value of $P_{1 s}$ with $P_{1 s}<P_{1 s v}$ we select the corresponding values of $K_{1 v}, K_{2 v}$ 
and $K_{v}$ to obtain three roots in Eq (27). Consequently, a set of values for $K_{1}, K_{2}$, $K$ and $P_{1 s}$ for each equilibrium curve with three different roots are obtained.

In Fig 3 a), a set of admissible values for $K_{1}, K_{2}$ and $K$ are plotted as a function of the pressure $P_{1 s}$, whereas an equilibrium curve with three roots in the movement zone of the mobile piston is shown in Fig 3 b) for a set of admissible values $K_{1}, K_{2}, K$ and $P_{l_{s}}$ that will be used hereafter (see table 1). Fig 4 shows a set of equilibrium curves which have been determined taking into account the previous steps and the slopes at the equilibrium points $P_{1}, P_{2}$ and $P_{3}$. It should be noted that the roots $d_{1 c}$ and $d_{3 c}$ associated to the points $P_{1}$ and $P_{3}$ are variable. This is important in the analysis of the system, since its behavior is very sensitive to the position of the equilibrium point because of the nonlinearity of the system equations (Eq. (20)) and the SRK and RK state equations (Eqs (12) and (13)).

Figure 3

Figure 4

Once the values of $K_{1}, K_{2}, K$ and $P_{1 s}$ have been determined, we shall analyze the stability of the equilibrium points $P_{1}, P_{2}$ and $P_{3}$ (see Fig 3 b)).

3.3 Stability of the equilibrium points of the mechanical subsystem

In order to analyze the stability of the equilibrium points of the mechanical subsystem, we shall rewrite Eq (26) as a system of two first-order differential equations. For the sake of simplicity in the notation, we first introduce the following parameters:

$$
p_{s}=\left(\frac{K_{1}}{m}+\frac{3 V_{s}^{2} K_{2}}{m S^{2}}-\frac{2 V_{s} K}{m S}\right) ; q_{s}=\left(\frac{3 V_{s} K_{2}}{m S^{2}}-\frac{K}{m S}\right) ; r_{s}=\frac{K_{2}}{m S^{2}}
$$

Taking into account Eqs (27) and (30), rewriting Eq (26) in term of the deviation volume $V_{p}^{\prime}(t)$ and introducing the variables $x_{1}(t)=V_{p}^{\prime}(t) ; x_{2}(t)=d V_{p}^{\prime}(t) / d t$ it is deduced that: 


$$
\left[\begin{array}{l}
\dot{x}_{1}(t) \\
\dot{x}_{2}(t)
\end{array}\right]=\left[\begin{array}{cc}
0 & 1 \\
-p_{s} & -\left(1-f_{p}\right) \frac{b}{m}
\end{array}\right]\left[\begin{array}{l}
x_{1}(t) \\
x_{2}(t)
\end{array}\right]+\left[\begin{array}{c}
0 \\
-q_{s} x_{1}^{2}(t)-r_{s} x_{1}^{3}(t)
\end{array}\right]
$$

On the other hand, considering the signs of the slopes at the equilibrium points $P_{1}, P_{2}$ and $P_{3}$ (see Fig 3b) and Fig 4) it can be easily demonstrated that the following inequalities are fulfilled:

$$
\left.\begin{array}{c}
\left(\frac{d y}{d V_{s}}\right)_{P_{1}}=\frac{3 K_{2}}{S^{2}} V_{s P_{1}}^{2}-\frac{2 K}{S} V_{s P_{1}}+K_{1}>0 ;\left(\frac{d y}{d V_{s}}\right)_{P_{3}}=\frac{3 K_{2}}{S^{2}} V_{s P_{3}}^{2}-\frac{2 K}{S} V_{s P_{3}}+K_{1}>0 \\
\left(\frac{d y}{d V_{s}}\right)_{P_{2}}=\frac{3 K_{2}}{S^{2}} V_{s P_{2}}^{2}-\frac{2 K}{S} V_{s P_{2}}+K_{1}<0
\end{array}\right\}
$$

where $V_{S P 1}, V_{S P 2}$ and $V_{S P 3}$ are the values of $V_{s}$ corresponding to the equilibrium points $P_{1}, P_{2}$ and $P_{3}$ respectively. From Eq (30) it is deduced that $p_{s}$ is a quadratic function of $V_{s}$ with a minimum at $V_{s m}=S K / 3 K_{2}$, and for this value we have that $m \cdot p_{s m}=\left(-K^{2}+3 K_{1} K_{2}\right) / 3 K_{2}$ is negative according to Eq (26). On the other hand, for $V_{s}=V_{s m}$ it is deduced that $q_{s}=0$, and therefore $q_{s}<0$ for $V_{s}<V_{s m}$ while $q_{s}>0$ for $V_{s}>V_{s m}$. Taking into account that the values of $V_{s}$ for which $p_{s}=0$ are given by $V_{s}=V_{s m}\left(1 \pm \sqrt{1-3 K_{1} K_{2} / K^{2}}\right)$, it follows that $p_{s}>0, q_{s}<0$ and $r_{s}>0$ at point $P_{l}$, whereas $p_{s}<0, q_{s}=0$ and $r_{s}>0$ at point $P_{2}$ and $p_{s}>0, q_{s}>0$ and $r_{s}>0$ at point $P_{3}$. According to the previous reasoning and taking into account Eq (32), the eigenvalues of the linear part of Eq (31) at points $P_{1}$ and $P_{3}$ are given by:

$$
\lambda_{1,2}=-\frac{1}{2}\left(1-f_{p}\right) \frac{b}{m} \pm \sqrt{\left(\frac{1-f_{p}}{2}\right)^{2} \frac{b^{2}}{m^{2}}-p_{s}^{2}}
$$

From Eq (33) it is deduced that for $f_{p}<1$ a stable focus (node) appears at $P_{1}$, whereas for $f_{p}>1$ an unstable focus (node) appears at $P_{3}$. On the other hand, the value $f_{p}=1$ leads to a pair of pure complex conjugate eigenvalues, for which Eq (32) allows to determine the integral curve as: 


$$
x_{2}^{2}=-p_{s} x_{1}^{2}-\frac{2}{3} q_{s} x_{1}^{3}-\frac{1}{2} r_{s} x_{1}^{4}+C
$$

where $\mathrm{C}$ is a constant which depends on the initial conditions. From a geometric viewpoint, the plot of $x_{2}$ versus $x_{1}$ obtained from $\mathrm{Eq}$ (34) is a closed curve which is symmetric with respect to the axis $x_{1}$, and therefore the system is periodic.

\subsection{Homoclinic orbits}

In accordance with the previous results, in this subsection we are going to investigate the conditions which lead to an homoclinic orbit when the values of $K_{1}, K_{2}$, $K$ and $P_{1 s}$ have be chosen to obtain three equilibrium points $P_{1}, P_{2}$ and $P_{3}$, being $P_{2}$ a saddle (see Fig 3 b), Fig 4 and Eq (33). Taking into account Eqs (20) and (25) and assuming that $f_{p}=1$ (i.e. the control law cancels the viscous friction and consequently $P_{1}$ and $P_{3}$ are weak focuses), the equations of the mechanical subsystem can be rewritten as follows:

$$
\begin{gathered}
\frac{d^{2} z(t)}{d t^{2}}=-p z(t)-q z^{2}(t)-r z^{3}(t)+I_{h} ; z(t)=V^{\prime}(t) \\
p=\frac{K_{1}}{m}+\frac{3 V_{s e}^{2} K_{2}}{m S^{2}}-\frac{2 V_{s e} K}{m S} ; q=\frac{3 V_{s e} K_{2}}{m S^{2}}-\frac{K}{m S} ; r=\frac{K_{2}}{m S^{2}} \\
I_{h}=-\frac{K_{1}}{m} V_{s e}-\frac{K_{2}}{m S^{2}} V_{s e}^{3}+\frac{K}{m S} V_{s e}^{2}+\frac{S^{2}}{m} P_{1 s}
\end{gathered}
$$

where for simplicity of notation the variable $z(t)$ has been introduced. Note that the signs of $p, q$ and $I_{h}$ will be positive or negative depending on the values of $K_{l}, K_{2}, K$, $P_{1 s}$. Therefore, we can choose different values for the previous magnitudes to determine the values of $V_{s e}$ for which $p, q$ and $I_{h}$ are positive as shown in Fig 5. It should be noted that there is a small range of values for $x_{s e}$ for which a homoclinic orbit can appear.

\section{Figure 5}

Introducing the coordinates $z_{1}(t), z_{2}(t)$ and considering the polynomial $P\left(z_{1}\right)$ whose intermediate root $z_{10}$ coincides with the horizontal coordinate of the saddle point $\mathrm{P}_{2}$ (see Figs $3 \mathrm{~b}$ ) and 4), Eq (35) can be rewritten in the form: 


$$
\left.\begin{array}{l}
\dot{z}_{1}(t)=z_{2}(t) \\
\dot{z}_{2}(t)=-p z_{1}(t)-q z_{1}^{2}(t)-r z_{1}^{3}(t)+I_{h}
\end{array}\right\} P\left(z_{1}\right)=-p z_{1}-q z_{1}^{2}-r z_{1}^{3}+I_{h}
$$

Eqs (36) can be integrated to yield:

$$
\left.\begin{array}{c}
\frac{1}{2} z_{2}^{2}=-\frac{1}{2} p z_{1}^{2}-\frac{1}{3} q z_{1}^{3}-\frac{1}{4} r z_{1}^{4}+I_{h} z_{1}+C \\
C=\frac{1}{2} p z_{10}^{2}+\frac{1}{3} q z_{10}^{3}+\frac{1}{4} r z_{10}^{4}-I_{h} z_{10}
\end{array}\right\}=\int_{z_{1 i}}^{t} d t
$$

where $z_{1 i}$ and $z_{1 f}$ are arbitrary values for the initial and final values of $z_{1}$ respectively. The polynomial inside the root of Eq (38) can be decomposed through direct polynomial division, so it holds that:

$$
\begin{gathered}
P_{1}\left(z_{1}\right)=-\frac{1}{2} r z_{1}^{4}-\frac{2}{3} q z_{1}^{3}-p z_{1}^{2}+2 I_{h} z_{1}+2 C=\left(z-z_{10}\right) P_{2}\left(z_{1}\right)+R \\
R=2 I_{h} z_{10}-p z_{10}^{2}-\frac{2}{3} q z_{10}^{3}-\frac{1}{2} r z_{10}^{4}-2 C \equiv 0
\end{gathered}
$$

where the rest $R$ of the polynomial division is zero in accordance with Eq (37), and therefore $z_{10}$ is root of the polynomial $P_{l}\left(z_{1}\right)$. In an analogous way, the polynomial $P_{2}\left(z_{1}\right)$ can be decomposed as:

$$
\begin{gathered}
P_{2}\left(z_{1}\right)=-\frac{1}{2} r z_{1}^{3}-\left(\frac{2}{3} q+\frac{1}{2} r z_{10}\right) z_{1}^{2}-\left(p+\frac{2}{3} q z_{10}+\frac{1}{2} r z_{10}^{2}\right) z_{1}+ \\
+\left[2 I_{h}-\left(p z_{10}+\frac{2}{3} q z_{10}^{2}+\frac{1}{2} r z_{10}^{3}\right)\right]=\left(z_{1}-z_{10}\right) P_{3}(z)
\end{gathered}
$$

where the polynomial $P_{3}(z)$ is defined as follows:

$$
\begin{gathered}
P_{3}(z)=-\frac{1}{2} r z_{1}^{2}-\left(r z_{10}+\frac{2}{3} q\right) z_{1}-\left(\frac{3}{2} r z_{10}^{2}+\frac{4}{3} q z_{10}+p\right)=a z_{1}^{2}+b z_{1}+c \\
P_{3}(z)=a z_{1}^{2}+b z_{1}+c=\left(z_{1}-\alpha_{1}\right)\left(z_{1}-\beta_{1}\right)
\end{gathered}
$$


being $\alpha_{1}$ and $\beta_{1}$ the smallest and greatest roots of the polynomial $P_{3}\left(z_{1}\right)$ respectively. Therefore, the integral given by Eq (38) can be developed through the expression:

$$
\int_{z_{1 i}}^{z_{1} f} \frac{d z_{1}}{\left(z_{1}-z_{10}\right) \sqrt{\left(z_{1}-\alpha_{1}\right)\left(z_{1}-\beta_{1}\right)}}=\int_{0}^{t} d t
$$

The results of the calculations in $\mathrm{Eq}(42)$ are the following ones:

- For the left lobe:

$$
z_{1 L}(t)=\frac{\beta_{1} \operatorname{th}^{2}(\gamma t / 2)+\alpha_{1} t_{1}^{2}}{\operatorname{th}^{2}(\gamma t / 2)+t_{1}^{2}} ; z_{2 L}(t)= \pm \frac{\gamma t_{1}^{2}\left(\beta_{1}-\alpha_{1}\right) \operatorname{th}(\gamma t / 2)}{\left\{\operatorname{ch}(\gamma t / 2)\left[\operatorname{th}^{2}(\gamma t / 2)+t_{1}^{2}\right]\right\}^{2}}
$$

- For the right lobe:

$$
z_{1 R}(t)=\frac{\beta_{1}+\alpha_{1} t_{1}^{2} \operatorname{th}^{2}(\gamma t / 2)}{1+t_{1}^{2} \operatorname{th}^{2}(\gamma t / 2)} ; z_{2 R}(t)= \pm \frac{\gamma t_{1}^{2}\left(\beta_{1}-\alpha_{1}\right) \operatorname{th}(\gamma t / 2)}{\left\{\operatorname{ch}(\gamma t / 2)\left[1+t_{1}^{2} \operatorname{th}^{2}(\gamma t / 2)\right]\right\}^{2}}
$$

where the parameters $\gamma$ and $t_{l}$ are given by:

$$
\gamma=\sqrt{\left(z_{10}-\alpha_{1}\right)\left(\beta_{1}-z_{10}\right)} ; t_{1}^{-1}=\sqrt{\frac{z_{10}-\alpha_{1}}{\beta_{1}-z_{10}}}
$$

By using the Eqs (43)-(45) we obtain several homoclinic orbits for the parameters values of $K_{1}, K_{2}, K$ and $P_{1 s}$ that appear in Figs 4 and 5, as it can be observed in Fig 6. The effect of such homoclinic orbits will be analyzed in connection with the chaotic behavior of the system.

\section{Figure 6}

3.5 Analysis of the oscillating motion

The results of the subsections 3.3 and 3.4 can be used to analyze the periodic orbits and to verify the previous analytical calculations as well as the adequate choice of the parameter values from a physical viewpoint. It should be recalled that points $P_{l}$ and 
$P_{3}$ are weak focuses, and according to Eqs (32)-(34) the piston has a periodic motion whose properties can be studied from Eqs (32). For the sake of notation simplicity, Eqs (32) can be written for $f_{p}=1$ as follows:

$$
\frac{d^{2} x(t)}{d t^{2}}+\omega^{2} x(t)=-\varepsilon q_{s} x^{2}(t)-\varepsilon r_{s} x^{3}(t) ; \omega^{2}=p_{s} ; x(t) \equiv x_{1}^{\prime}(t)
$$

where the parameter $\varepsilon$ is considered to be small in order to apply the successive approximations method based on the Laplace transform [15]. Essentially, we are assuming that the function $-q_{s} x^{2}(t)-r_{s} x^{3}(t)$ represents a perturbation so that for $\varepsilon=0$ $\mathrm{Eq}$ (46) is reduced to a harmonic oscillator, whereas for $\varepsilon=1 \mathrm{Eq}$ (46) reduces to the equation whose approximate solution we want to obtain. We will assume that the independent variable $x(t)$ and the frequency $\omega^{2}$ can be expanded as follows:

$$
\left.\begin{array}{c}
x(t)=x_{1}(t)+\varepsilon x_{2}(t)+\varepsilon^{2} x_{3}(t)+\ldots . . \\
\omega^{2}=\omega_{1}^{2}+\varepsilon C_{2}+\varepsilon^{2} C_{3}+\ldots \ldots .
\end{array}\right\}
$$

where the constants $\omega_{1}, C_{2}, C_{3} \ldots$ must be calculated in the recursive process. Substituting Eqs (47) into Eq (46) and taking Laplace transform (denoted by $\boldsymbol{L}$ and denoting by $\mathrm{s}$ the Laplace transform variable) in Eq (47) with initial conditions $x(0)=A_{o} ; d x(0) / d t=0$, the following set of recursive equations are deduced:

$$
\begin{aligned}
& \varepsilon^{0} \rightarrow\left(s^{2}+\omega_{1}^{2}\right) X_{1}(s)=s A_{0} \\
& \varepsilon^{1} \rightarrow\left(s^{2}+\omega_{1}^{2}\right) X_{2}(s)=-C_{2} X_{1}(s)-q_{s} \boldsymbol{L}\left[x_{1}^{2}(t)\right]-r_{s} \boldsymbol{L}\left[x_{1}^{3}(t)\right] \\
& \varepsilon^{2} \rightarrow\left(s^{2}+\omega_{1}^{2}\right) X_{3}(s)=-C_{3} X_{1}(s)-C_{2} X_{2}(s)-2 q_{s} \boldsymbol{L}\left[x_{1}(t) x_{2}(t)\right]-3 r_{s} \boldsymbol{L}\left[x_{l}^{2}(t) x_{2}(t)\right]
\end{aligned}
$$

The first order approximation is simply the cosine function $x_{1}(t)=A_{0} \cos \omega_{1} t$, from which the second Eq (48) allows to obtain its Laplace transform $X_{2}(s)$. Then the inverse Laplace transform of $X_{2}(s)$ provides the second order approximation $x_{2}(t)$ as: 


$$
\begin{aligned}
x_{2}(t) & =-\frac{C_{2} A_{0}}{2 \omega_{1}} t \sin \omega_{1} t+\frac{q_{s} A_{0}^{2}}{6 \omega_{1}^{2}} \cos 2 \omega_{1} t+\frac{q_{s} A_{0}^{2}}{3 \omega_{1}^{2}} \cos \omega_{1} t-\frac{q_{s} A_{0}^{2}}{2 \omega_{1}^{2}}- \\
& -\frac{3 r_{s} A_{0}^{3}}{8 \omega_{1}^{2}} t \sin \omega_{1} t+\frac{r_{s} A_{0}^{3}}{32 \omega_{1}^{2}} \cos 3 \omega_{1} t-\frac{r_{s} A_{0}^{3}}{32 \omega_{1}^{2}} \cos \omega_{1} t
\end{aligned}
$$

Since the solution is periodic, the non-periodic terms in $t \sin \omega_{1} t$ must be cancelled, and therefore the constants $C_{2}$ and $\omega_{1}$ can be determined. Besides, the second order approximation is known from Eq (49), so we obtain that:

$$
C_{2}=-\frac{3 r_{s} A_{0}^{2}}{4} ; \omega_{1}=\sqrt{\omega^{2}+\frac{3 r_{s} A_{0}^{2}}{4}}
$$

Although the calculations are somewhat cumbersome, the third Eq (48) allows to obtain the third-order approximation as:

$$
\begin{gathered}
C_{3}=-\frac{47 r_{s}^{2} A_{0}^{4}}{256 \omega_{1}^{2}}+\frac{q_{s} r_{s} A_{0}^{3}}{4 \omega_{1}^{2}}-\frac{5 q_{s}^{2} A_{0}^{2}}{12 \omega_{1}^{2}}=-\frac{C}{\omega_{1}^{2}} \\
\omega_{1}=\sqrt{\frac{1}{2}\left(\omega^{2}+\frac{3}{4} r_{s} A_{0}^{2}\right)+\sqrt{\frac{1}{4}\left(\omega^{2}+\frac{3}{4} r_{s} A_{0}^{2}\right)^{2}+C}}
\end{gathered}
$$

The expression for the third-order approximation $x_{3}(t)$ is similar to the one of Eq (49) but with eleven terms, as it is shown in the Appendix. This approximate analytical solution $x_{3}(t)$ shows that the periodic motions are stable. To corroborate this issue, the values of $p_{s}$ and $q_{s}$ in Eq (31) have been obtained for the weak focuses $P_{1}$ and $P_{3}$ (equilibrium points in Fig 3 b)). Fig 7 a) depicts two integration curves in the phase plane $x_{p}(t)-\dot{x}_{p}(t)$, where $x_{p}(t)$ denotes the piston position. It should be noted that these curves are closed around the equilibrium points $P_{1}$ and $P_{3}$ in accordance with Eq (34). This behavior is only possible in the zones where $p_{s}>0$, according to the previous discussion regarding the sign of $p_{s}, q_{s}$ and $r_{s}$ (see Eq (31)). Thus it is impossible to obtain an oscillating motion for piston positions such that $p_{s}<0$.

Fig 7 b) depicts the homoclinic orbit for the parameter values indicated in Table 1 together with the two periodic orbits plotted in Fig 7 a). The parameter values are $p_{s 1}$ 
$=55.5681, q_{s 1}=-3.8683 \cdot 10^{4}, r_{s 1}=6.1097 \cdot 10^{6}$ for point $P_{1}$ and $p_{s 3}=48.6927, q_{s 3}=$ $3.7020 \cdot 10^{4}, r_{s 3}=6.1087 \cdot 10^{6}$ for point $P_{3}$.

On the other hand, Fig 7 c) shows the exact temporal response of the mechanical subsystem and the approximate one deduced from Eqs (A6)-(A9) (see the Appendix) assuming an initial amplitude of $0.0388 \mathrm{~m}$ for the equilibrium point $P_{1}$. It is clear that the exact and approximate simulation results are very close, and indeed the exact simulation period is $0.9225 \mathrm{~s}$ whereas the approximate one deduced from Eq (51) is $0.9087 \mathrm{~s}$, i.e. with a relative error of $1.5 \%$.

To conclude this section, Fig 7 d) shows the case of the equilibrium point $P_{3}$ assuming an initial amplitude of $0.0648 \mathrm{~m}$. The exact simulation period is $0.8935 \mathrm{~s}$ and the approximate one is $0.8559 \mathrm{~s}$, i.e. with and error of $4.2 \%$. Although the approximation is still reasonable, a more accurate result could be obtained by considering the fourth-order approximation.

Figure 7

\section{Analysis of the steady-state and oscillating behavior of the system}

Once the parameter values have been determined and the structure of control system has been defined, we are going to analyze the behavior of the whole system given by Eqs (20) and (24) taking into account the SRK and RK state equations given by Eqs (12) and (13) respectively. However, before carrying out this study it is necessary to determine the specific heat for the nitrogen at constant volume $c_{v}(T)$ in Eqs (20). For this purpose, the specific heat of the nitrogen at constant pressure $c_{p}(T)$ can be obtained at low pressure values as [29]:

$$
c_{p}(T)=29.342-3.5395 .10^{-3} T+1.007 .10^{-5} T^{2}-4.3116 .10^{-9} T^{3}+2.5935 .10^{-13} T^{4}
$$

where $T$ is the temperature (in $K)$ and $c_{p}(T)$ is expressed with units of Joules/(mole $K$ ). Once the specific heat at constant pressure $c_{p}(T)$ is known, the specific heat at constant volume $c_{v}(T)$ is obtained as [1-5] [28], [30], [31]: 


$$
c_{p}-c_{v}=-T\left(\frac{\partial P}{\partial v}\right)_{T} \cdot\left(\frac{\partial v}{\partial T}\right)_{P}^{2}=\frac{T v \beta^{2}}{\kappa}
$$

where $\beta$ is the coefficient of isobaric expansion and $\kappa$ is the coefficient of isothermal compressibility, which are defined as follows:

$$
\beta=\frac{1}{v}\left(\frac{\partial v}{\partial T}\right)_{P} ; \kappa=-\frac{1}{v}\left(\frac{\partial v}{\partial P}\right)_{T}
$$

The coefficients $\beta$ and $\kappa$ can be obtained from the state equations given by Eqs (12) and (13). Therefore, Eqs (52) and (53) allow to determine the specific heat at constant volume which appears in Eqs (20). On the other hand, since the values of $c_{p}(T)$ given in $\mathrm{Eq}$ (52) are determined at low pressure, it is necessary calculate the variation of $c_{p}(T)$ with respect to the pressure by means of the following equation [4-5], [28], [30]:

$$
\Delta c_{p}=-\int_{P_{1}}^{P_{2}} T\left(\frac{\partial^{2} v}{\partial T^{2}}\right)_{P} d P
$$

where $P_{1}$ is a low pressure value (i.e. between 0.5 and $1 \mathrm{~atm}$ ) and $P_{2}$ is the nitrogen pressure in the cylinder at each time instant. Since the gas behavior is very sensitive to small changes in any of the state magnitudes $P, v$ and $T$, to avoid numerical problems in the simulation process it is convenient to carry out the calculations as follows:

i) For the SRK state equation:

The value of $(\partial v / \partial T)_{P}$ is deduced from Eq (12) through the derivate at constant pressure, so it follows that:

$$
\left(\frac{\partial v}{\partial T}\right)_{P}=\frac{R-\frac{a(\partial \bar{\alpha} / \partial T)(v-b)}{v(v+b)}}{\frac{R T}{v-b}-\frac{a \bar{\alpha}(2 v+b)(v-b)}{v^{2}(v+b)^{2}}}=\frac{R v(v+b)-a(\partial \bar{\alpha} / \partial T)(v-b)}{P v(v+b)-a \bar{\alpha}\left[\frac{v^{2}-2 b v-b^{2}}{v(v+b)}\right]}=\frac{f_{1}(v)}{f_{2}(v)}
$$


The variation of the specific heat at constant pressure with respect to the pressure can be calculated from Eqs (55) and (56) as follows:

$$
\Delta c_{p}(T)=-\int_{P_{1}}^{P_{2}} T\left[\frac{\left(\partial f_{1}(v) / \partial v\right) f_{2}(v)-f_{1}(v)\left(\partial f_{2}(v) / \partial v\right)}{f_{2}^{2}(v)}\right]\left(\frac{\partial v}{\partial T}\right)_{P} d P
$$

On the other hand, it should be taken into account that:

$$
\left(\frac{\partial P}{\partial v}\right)_{T}=-\frac{R T}{(v-b)^{2}}+\frac{a \bar{\alpha}(2 v+b)}{v^{2}(v+b)^{2}}
$$

Eqs (56) and (58) provide the values of $\beta$ and $\kappa$ in Eqs (54), and therefore the specific heat at constant volume and at high pressure can be estimated from Eqs (53) and (57).

ii) For the RK state equation:

The calculations are similar to the previous case, and the variation of the volume with respect to temperature at constant pressure is given by:

$$
\left(\frac{\partial v}{\partial T}\right)_{P}=\frac{R+\frac{(1 / 2) a T^{-3 / 2}(v-b)}{v(v+b)}}{\frac{R T}{v-b}-\frac{a T^{-1 / 2}(2 v+b)(v-b)}{v^{2}(v+b)^{2}}}=\frac{R v(v+b)+(1 / 2) a T^{-3 / 2}(v-b)}{P v(v+b)-a T^{-1 / 2}\left[\frac{v^{2}-2 b v-b^{2}}{v(v+b)}\right]}=\frac{f_{1}(v)}{f_{2}(v)}
$$

The previous equation can be applied taking into account the RK state equation to obtain the specific heat at constant volume. The simulation process is carried out as follows:

- We take $T_{s}=200 \mathrm{~K}$ as the set point temperature and an initial pressure of $P_{s}=$ $40 \mathrm{~atm}$, which are far away from the critical point $\left(T_{c}=126.1 \mathrm{~K}, P_{c}=33.94\right.$ atm).

- We choose the initial conditions by using the deviation variables stated in Eqs (20) and (24), like for instance $x_{0}=\left[0.01 V_{s e} 00.01 * T s 0\right]$. The value of the heat 
in steady state is then calculated from the flow rate of the heating fluid $Q_{s}=K_{s} F_{s e}$ with $F_{s e}=8.9771 .10^{-6} \mathrm{~m}^{3} / \mathrm{s}$ and $Q_{s}=6.8525 \mathrm{~kJ} / \mathrm{s}$.

- With the previous values of $T_{s}, P_{s}$, and $V_{s}=0.006 \mathrm{~m}^{3}$ (initial gas volume), the mole number $n$ is determined from the SRK and RK state equations.

In each simulation step, the molar volume is calculated from the value of $V^{\prime}(t)$ (Eq (20)) as follows: $v(t)=1000\left(V^{\prime}(t)+V_{\text {se }}\right) / n$ (liters/mole). The pressure is calculated with the temperature $T^{\prime}(t)+T_{s}$ obtained in the previous iteration. Next, the compressibility coefficient $z(t)=P(t) v(t) / R T(t)$ and the piston displacement $x_{p}(t)=V^{\prime}(t)+V_{s e}+V_{c}+V_{s d} / S$ are calculated.

- The values of $\beta$ and $\kappa$ are calculated from Eqs (54). Next, from Eqs (56), (57) and (53) the specific heat at constant volume is evaluated, and therefore we have all the required magnitudes to determine the state variables of $\mathrm{Eq}(20)$ for the next iteration.

Fig 8 depicts the simulation results, which have been obtained by using a fourthorder Runge-Kutta integration scheme with a simulation step of $0.005 \mathrm{~s}$. The set point of the piston position is the corresponding one to point $P_{3}$ (see Figs $3 \mathrm{~b}$ ) and $7 \mathrm{~b}$ )), and the set point temperature is $T_{s}=200 \mathrm{~K}$. In addition, it is assumed that $f_{p}=0$ in the control law given by Eq (24), i.e. the friction effect is considered.

Fig 8 a) shows that the set point is reached after $10 \mathrm{~s}$ according to the results given in Eqs (32) and (33). Besides, Fig 8 b) depicts the plots of the gas temperatures $T_{g l}(t)$ and $T_{g_{2}}(t)$ obtained from the SRK and RK state equations respectively. It should be noted that the gas temperature remains with very small oscillations around the desired set point due to the action of the PI controller (see Fig 1). Consequently, the process can be regarded as almost isotherm from a thermodynamic viewpoint. In addition, in Fig 8 b) an approximate solution of the system temperature $T_{a}(t)$ assuming the SRK state equation is plotted. This approximated solution has been obtained as it is explained below.

It should be recalled that when $d v^{\prime} / d t \approx 0$ (i.e. when the system reaches a behavior close to the steady state), Eq (17) can be rewritten as follows: 


$$
\frac{d T^{\prime}(t)}{d t} \approx-\frac{\beta K_{s}}{n} T^{\prime}(t)+\frac{\alpha K_{s}}{n} Q^{\prime}(t)+\frac{Q^{\prime}(t)}{n c_{v m}}+\frac{T^{\prime}(t)}{\left[1+Q^{\prime}(t) / Q_{s}\right]}\left[\frac{\beta K_{s}}{n}+\frac{\beta}{\alpha n c_{v m}}\right]
$$

where $c_{v m}$ is the constant mean value of the specific heat at constant volume. Expanding on the term $1 / 1+Q^{\prime}(t) / Q_{s}$ and assuming that the product of the deviation variables $T^{\prime}(t) Q^{\prime}(t)$ is small, Eq (60) can be rewritten in linear form to yield:

$$
\frac{d T^{\prime}(t)}{d t} \approx \frac{\beta}{n \alpha c_{v m}} T^{\prime}(t)+\left(\frac{\alpha K_{s}}{n}+\frac{1}{n c_{v m}}\right) Q^{\prime}(t)
$$

Eliminating $Q^{\prime}(t)$ between Eqs (18) and (61) we obtain a second order linear differential equation, which expressed in terms of the absolute variable $T(t)\left(T^{\prime}(t)=T(t)\right.$ - $T_{s}$ ) can be integrated from initial conditions $T\left(t_{i}\right) ; d T\left(t_{i}\right) / d t$ to give:

$$
\begin{gathered}
T_{a}(t)=T\left(t_{i}\right)+\frac{1}{\omega_{n} \sqrt{1-\delta^{2}}} \frac{d T\left(t_{i}\right)}{d t} \exp \left(-\delta \omega_{n} t\right) \sin \left[\omega_{n} \sqrt{1-\delta^{2}} t-\varphi\right] \\
2 \delta \omega_{n}=K_{s} K_{p}\left(\frac{\alpha K_{s}}{n}+\frac{1}{n c_{v m}}\right)-\frac{\beta}{n \alpha c_{v m}} ; \omega_{n}=\sqrt{\frac{K_{s} K_{p}}{\tau_{i}}\left(\frac{\alpha K_{s}}{n}+\frac{1}{n c_{v m}}\right)} \\
\varphi=\omega_{n} \sqrt{1-\delta^{2}} t_{i} ; \text { for } t \geq t_{i}
\end{gathered}
$$

where the phase angle $\varphi$ depends on the initial time $t_{i}$ for which the approximation given by Eqs (62) is obtained. The results of Fig $8 \mathrm{~b}$ ) indicate that $T_{a}(t)$ is close to the temperature $T_{g l}(t)$ obtained with the SRK state equation. In addition, the previous reasoning shows why the behavior of the system is oscillating with decreasing amplitude. In Figs $8 \mathrm{c}$ ) and $8 \mathrm{~d}$ ), the heat generated in the heating coils and the respective pressures for the SRK and RK state equations are plotted showing that the results are very similar for the generated heat, but that there is a small divergence in the pressure (in steady state, the SRK equation leads to $40 \mathrm{~atm}$ whereas the RK equation leads to $39.5 \mathrm{~atm}$ ). Thus we have demonstrated that both the mechanical and thermal subsystems are suitably controlled.

Figure 8 
To corroborate the calculations from a thermodynamic viewpoint, Fig 9 a) shows the specific heats at constant pressure and volume for the SRK and RK state equations (see Eqs (52)-(59)). It should be noted that there are differences between the two state equations, especially with regard to the specific heat at constant volume. Nevertheless, the well known inequality $c_{p}>c_{v}$ of Thermodynamics is fulfilled for both state equations.

Fig 9 b) shows the variation of the compressibility coefficient. It has been corroborated that the values for $z$ obtained from the SRK equation are closer to the experimental values reported for the nitrogen than the ones for the RK equation [5], [28], [30-31]. On the other hand, Fig 9 c) shows the function given by:

$$
F_{1} F_{2} F_{3}=\left(\frac{\partial P}{\partial v}\right)_{T}\left(\frac{\partial v}{\partial T}\right)_{P}\left(\frac{\partial T}{\partial P}\right)_{v}=-1
$$

It is well known that a system is in thermodynamic equilibrium [1-5] [28], [3031] if Eq (63) is fulfilled. To analyze this issue for the SRK and RK state equations, the product $F_{1} F_{2} F_{3}$ has been plotted as a function of the time in Fig $9 \mathrm{c}$ ). It is interesting to remark that the SRK state equation leads exactly to $F_{1} F_{2} F_{3}=-1$, whereas for the RK state equation $F_{1} F_{2} F_{3}$ remains oscillating around -0.86 . Therefore it is possible to ensure that the simulation results obtained from the SRK state equation represent better the thermodynamic process than the ones for RK state equation. Additionally, $F_{1}, F_{2}$ and $F_{3}$ are separately plotted in Fig 9 d) to emphasize that their values are quite far away from unity, which does not contradict the fact that their product is exactly equal to -1 in the case of the SRK state equation.

\section{Figure 9}

Fig 10 shows an example of oscillating behavior obtained from the analysis carried out in subsection 3.5. Fig 10 a) shows the piston motion, whose angular frequency is close to the one obtained from Eq (51). Besides, Fig $10 \mathrm{~b}$ ) shows that the temperature (for the SRK and RK state equations) remains with oscillations around the set point $T_{s}=200 \mathrm{~K}$ due to the PI control action, whereas Fig $10 \mathrm{c}$ ) shows an oscillating behavior for the heat generated in the heating coils. Finally, the product of the functions 
$F_{1}, F_{2}$ and $F_{3}$ given by Eq (63) is plotted as a function of time in Fig $10 \mathrm{~d}$ ). As in the previous case, the SRK state equation leads exactly to $F_{1} F_{2} F_{3}=-1$, and the same considerations of Figs 9 c) and d) can be made.

\section{Figure 10}

\section{Chaotic behavior of the system}

The purpose of this section is to investigate the conditions which lead to chaotic motions of the piston and how this behavior can affect the time evolution of the thermal variables. Additionally, the fulfillment of thermodynamic equilibrium condition given by Eq (63) will be analyzed in the presence of chaos assuming the SRK and RK state equations. For this purpose, the calculation of the homoclinic orbits carried out in subsection 3.4 will be used to apply the Melnikov method [22-27] with the aim of obtaining sufficient conditions for chaotic behavior.

It should be recalled that the homoclinic orbits for the mechanical subsystem are known according to Eqs (43)-(45), whereas the control law given by Eq (24) implies a decoupling between the mechanical and thermal subsystems. Consequently, considering a small harmonic disturbance on the control law which acts over the piston motion and assuming that there is friction, Eqs (36) can be rewritten as follows:

$$
\left.\begin{array}{l}
\dot{z}_{1}(t)=z_{2}(t) \\
\dot{z}_{2}(t)=-p z_{1}(t)-q z_{1}^{2}(t)-r z_{1}^{3}(t)+I_{h}+\varepsilon\left[A_{p} \sin \omega_{p} t-\delta z_{2}(t)\right]
\end{array}\right\}
$$

where $\delta=b / m$ whereas $\varepsilon>0$ is a small perturbation parameter to account for the effect of the harmonic disturbance and the friction effect. From Eqs (43) and (64), the Melnikov function for the left lobe of the homoclinic orbit (see Figs 6 and 7) can be written as follows:

$$
\begin{gathered}
M_{L}\left(t_{0}\right)=A_{p} \cos \omega_{p} t_{0} \cdot I_{1}-\delta I_{2} \\
I_{1}=\int_{-\infty}^{\infty} \frac{s h(\gamma t / 2) \sin \omega_{p} t}{c h^{3}(\gamma t / 2)\left[t h^{2}(\gamma t / 2)+t_{1}^{2}\right]} d t ; I_{2}=\int_{-\infty}^{\infty}\left\{\frac{s h(\gamma t / 2) \sin \omega_{p} t}{c h^{3}(\gamma t / 2)\left[t h^{2}(\gamma t / 2)+t_{1}^{2}\right]}\right\}^{2} d t
\end{gathered}
$$


Similar expressions to the ones of Eq (65) can be obtained for the right lobe. Next we obtain Melnikov's functions (with zeros) for different values of $A_{p}$ and $\omega_{p}$, and the combination of these values provides a sufficient condition for chaotic behavior [2627], [32] as shown in Fig 11.

Fig 11 a) depicts the curve $A_{p}=\delta I_{2} / I_{1}$ plotted as a function of the disturbance frequency $\omega_{p}$. It is interesting to remark that a combination of values $\left(\omega_{p}, A_{p}\right)$ below or at the curve cannot lead to chaotic behavior, so we can ensure that chaos does not appear with the values indicated at points $P_{1}$ and $P_{2}$. However, point $P_{3}$ may lead to chaotic behavior. Fig 11 b) shows several Melnikov functions which have been obtained for the following values:

$$
\left[\begin{array}{l}
A_{p} \\
\omega_{p}
\end{array}\right]=\left[\begin{array}{ccccc}
2.0 \cdot 10^{-3} & 1.15 \cdot 10^{-2} & 2.1 \cdot 10^{-2} & 3.05 \cdot 10^{-2} & 4.0 \cdot 10^{-2} \\
1 & 3.25 & 5.5 & 7.75 & 10
\end{array}\right]
$$

where $A_{p}$ and $\omega_{p}$ have units of $\mathrm{m}^{3} / \mathrm{s}^{2}$ and $\mathrm{rad} / \mathrm{s}$ respectively. It should be noted that the Melnikov function has no zeros for $A_{p}=2 \cdot 10^{-3}$ and $A_{p}=1.15 \cdot 10^{-2}$, so the chaotic motion cannot appear for such values.

\section{Figure 11}

Fig 12 shows the time evolution of the pressures, specific volumes, temperatures and compressibility coefficients obtained with the SRK and RK state equations with $A_{p}$ $=0.02 \mathrm{~m}^{3} / \mathrm{s}^{2}$ and $\omega_{p}=5.2 \mathrm{rad} / \mathrm{s}$ (which according to Fig 11 are in the potential chaotic zone). The initial conditions and simulation values are the same as the ones indicated in the previous section. It should be noted that the behaviors of the pressure, specific volume, the temperatures and compressibility coefficient in Figs 12 a), b) c) and d) seem to be chaotic, although the temperatures remain with small oscillations around the set point due to the PI control action. On the other hand, the pressure values of Fig 12 a) are greater than pressures in steady state and in oscillating regime shown in Fig 8 d). It is important to remark that the differences between the values of the state variables shown of Fig 12 are simply due to the fact that they have been calculated with the SRK and RK state equations, which provide different results. 
To elucidate whether the results of Fig 12 are chaotic, we are going to calculate the sensitive dependence when the system is simulated with two very close initial conditions. Besides, we shall calculate the power spectral density and all the Lyapunov exponents of the system given by Eqs (12), (20) and (24) with $f_{p}=0$ (to account for the friction effect). The power spectral density is calculated from the Fourier transform [32], and for the Lyapunov exponents we consider the following vector field:

$$
\left.\begin{array}{c}
f_{1}=\frac{d x_{1}(t)}{d t}=x_{2}(t) \\
f_{2}=-\frac{K_{1}}{m}\left[x_{1}(t)+V_{s e}\right]-\frac{K_{2}}{m S^{2}}\left[x_{1}(t)+V_{s e}\right]^{3}-\frac{b}{m} x_{2}(t)+\frac{K}{m S}\left[x_{1}(t)+V_{s e}\right]^{2}+A_{p} \sin \left(\omega_{p} t\right) \\
f_{3}=-\frac{\beta K_{s}}{n} x_{3}(t)+\frac{\alpha K_{s}}{n} x_{4}(t)+\left[K_{s}+\frac{1}{\alpha c_{v}(t)}\right] \frac{\beta Q_{s} x_{3}(t)}{n\left[x_{4}(t)+Q_{s}\right]}+ \\
+\frac{x_{4}(t)}{n c_{v}(t)}-\left[\frac{x_{3}(t)+T_{s}}{n c_{v}(t)}\right]\left(\frac{\partial P}{\partial T}\right)_{v} x_{2}(t) ; \quad f_{4}=-K_{s} K_{p} f_{3}-\frac{K_{s} K_{p}}{\tau_{i}} x_{3}
\end{array}\right\}
$$

being:

$$
\left.\begin{array}{c}
{\left[x_{1}(t), x_{2}(t), x_{3}(t), x_{4}(t)\right] \equiv\left[V^{\prime}(t), \dot{V}^{\prime}(t), T^{\prime}(t), Q^{\prime}(t)\right]} \\
\left(\frac{\partial P}{\partial T}\right)_{v}=\frac{R}{v(t)-b}-\frac{m_{i} a \bar{\alpha}^{1 / 2}}{v(t)(v(t)+b)\left[\left(x_{3}(t)+T_{s}\right) \cdot T_{c}\right]^{1 / 2}} ; v(t)=\frac{1000}{n}\left[x_{1}(t)+V_{s e}\right]
\end{array}\right\}
$$

where $v$ is the molar volume in liters/mol and $x_{1}(t)$ is the deviation volume in $m^{3}$. Now it is necessary to linearize the vector field given by Eqs (67) throughout a trajectory as well as to apply the Gram-Smidth orthogonalization method [32], [36]. Since Eqs (67) are not autonomous, for numerical purposes it is convenient to transform it into an autonomous by introducing the variable $x_{5}(t)=\omega_{p} t$, which implies that $\dot{x}_{5}(t)=\omega_{p}$. A way to check the correctness of the results is to solve the system of equations (67) and (68) with the purpose of checking that the sum of Lyapunov exponents approximately coincides with average of the vector field divergence. For this purpose, the Runge-Kutta and Runge-Kutta-Felhberg methods have been used both with a simulation step of 0.002 
s. Both methods have provided almost the same results, which is an indicator of the correctness of the numerical simulations.

Fig 13 a) depicts two simulations $x_{p}(t)$ and $x_{p d}(t)$ of the piston position assuming the SRK state equation for two very close initial conditions. It should be noted that from approximately $t=23 \mathrm{~s}$ the oscillations are completely different, and thus the system exhibits sensitive dependence. In Fig $13 \mathrm{~b}$ ) the error defined as $E=x_{p}(t)-x_{p d}(t)$ is plotted as a function of the time, once again showing sensitive dependence as a clear indicator of chaos.

In Fig 13 c) the Lyapunov exponents of the whole system have been plotted showing that there are two positive exponents, two negative ones and one null exponent (the one associated to the oscillation variable $x_{5}(t)$ ). This result is a clear indicator that the oscillations shown in Fig 12 and Fig 13 a) are chaotic. Furthermore, it should be noted that it is not very frequent to obtain two positive Lyapunov exponents (hyperchaotic system), since the most common situation in chaotic systems is to obtain a single positive Lyapunov exponent. The values of the Lyapunov exponents at $t=150$ $s$ are the following ones:

$$
L Y=\left[\begin{array}{lllll}
1.031587 & 0.020587 & -0.047190 & -1.848809 & 0
\end{array}\right]
$$

Fig 13 d) shows the sum of the Lyapunov exponents together with the divergence of the vector field given by Eqs (67). It is interesting to remark that after a short transient the sum of the Lyapunov exponents remains approximately constant and equal to a value of -0.84382 , around which the divergence of the vector field remains oscillating with small oscillations. Thus the following approximation is fulfilled:

$$
\sum_{i=1}^{5} L Y_{i} \approx \operatorname{div} \bar{f}
$$

To verify that the approximation of equation (70) is fulfilled, the relative error between $\sum_{i=1}^{5} L Y_{i}$ and $\operatorname{divf}$ is defined as: 


$$
\operatorname{Er}(t)=\frac{\sum_{i=1}^{5} L Y_{i}\left(t_{m}\right)-\frac{1}{t-t_{1}} \int_{t_{1}}^{t} \operatorname{div}(\vec{f}) d t}{\sum_{i=1}^{5} L Y_{i}\left(t_{m}\right)} \text { for } t<t_{m}
$$

where $t_{m}$ is the simulation time, $\vec{f}$ is the vector field whose components are given by Eqs (67) and (68) and $t_{1}$ is a time which is arbitrarily selected when the transitory state in the numerical computation becomes small. The previous equation has been implanted with the simulation results that arise from the Runge-Kutta and Runge-Kutta-Felhberg integration methods for a simulation step of $T=0.002 \mathrm{~s}$, and both methods lead to an average relative error of approximately $0.18 \%$. This is another indicator of the correctness of the simulation results.

Figure 13

In Fig 14 a) the power spectral density for the gas volume calculated through the SRK state equation shows a broadband spectrum with decaying oscillations, which is a characteristic feature of a chaotic signal [32]. In addition, the peak of the applied external disturbance with $\omega_{p}=5.2 \mathrm{rad} / \mathrm{s}$ can be clearly distinguished within the spectrum. Fig $14 \mathrm{~b}$ ) shows the specific heats $c_{p}$ at constant pressure calculated for the SRK and RK state equations and the corresponding specific heat $c_{v}$ at constant volume. As in the case of steady state behavior indicated in Fig 9 a), the thermodynamic inequality $c_{p}>c_{v}$ is fulfilled. On the other hand, the product $F_{1} \cdot F_{2} \cdot F_{3}$ given by Eq (63) is shown in Fig $14 \mathrm{c}$ ). Like in the previous cases of steady state (Fig $9 \mathrm{c}$ )) and oscillating behavior (Fig $10 \mathrm{~d}$ )), $F_{1} \cdot F_{2} \cdot F_{3}$ is exactly equal to -1 for the SRK state equation, whereas it remains oscillating around -0.86 for the RK state equation. Fig $14 \mathrm{~d}$ ) shows the plot of the function $F_{1} \cdot F_{2} \cdot F_{3}+1$ for the SRK state equation to remark that the relation $F_{1} \cdot F_{2} \cdot F_{3}$ $+1=0$ is fulfilled with a very low error, which is exclusively due to the computational calculation.

Figure 14

Figs 15 a) and b) show two strange attractors, where it has been assumed that the piston position is constrained between $0.18 \mathrm{~m}$ and $0.9 \mathrm{~m}$. In Fig $15 \mathrm{a}$ ) we can clearly 
appreciate the presence of two attraction basins as well as how the homoclinic orbit is destroyed due to the harmonic disturbance on the piston motion. On the other hand, Fig $15 \mathrm{~b})$ shows that the control force applied to the piston can be regarded as physically feasible, in accordance with the discussion of the mechanical parameter values of section 3 .

\section{Figure 15}

5.1 Procedure to obtain a desired gas behavior from a chaotic regime

In this section we demonstrate the possibility of changing the controller structure so that the chaotic behavior is destroyed at a certain time instant, after which the gas temperature and pressure will reach a prescribed behavior. For this purpose, the following two hypotheses will be assumed: i) The gas is outside of the biphasic zone at sufficiently high pressures and low temperatures above the critical point. ii) Only the Soave-Redlich-Kwong state equation will be considered.

In order to modify controller, for $t \geq t_{a}$ the control force applied to the mobile piston given in the first Eq of (20) is changed to:

$$
\begin{gathered}
\frac{S}{m}\left[F^{\prime}(t)+F_{s}\right]=\frac{S^{2}}{m}\left[P^{\prime}(t)+P_{s}\right]-\frac{b}{m} \frac{d V^{\prime}(t)}{d t}-\frac{K_{1}}{m}\left[V^{\prime}(t)+V_{s e}\right]-\frac{K_{2}}{m S^{2}}\left[V^{\prime}(t)+V_{s e}\right] \\
+\omega_{n F}^{2}\left[V^{\prime}(t)-V_{d}^{\prime}(t)\right]+2 \delta_{F} \omega_{n F} \frac{d\left[V^{\prime}(t)-V_{d}^{\prime}(t)\right]}{d t}-\frac{d^{2} V_{d}^{\prime}(t)}{d t^{2}}
\end{gathered}
$$

where $V_{d}^{\prime}(t)$ is a prescribed volume for the gas, $\omega_{n F}$ is a natural oscillation frequency and $\delta_{F}$ is a damping coefficient such that $0<\delta_{F}<1$. Substituting Eq (72) into the first Eq of (20) it is deduced that:

$$
\frac{d^{2} V^{\prime}(t)}{d t^{2}}=-2 \delta_{F} \omega_{n F} \frac{d\left[V^{\prime}(t)-V_{d}^{\prime}(t)\right]}{d t}-\omega_{n F}^{2}\left[V^{\prime}(t)-V_{d}^{\prime}(t)\right]+\frac{d^{2} V_{d}^{\prime}(t)}{d t^{2}}
$$

Introducing the error variable $e_{V}(t)=V^{\prime}(t)-V_{d}^{\prime}(t), \mathrm{Eq}(73)$ and its solution can be expressed as: 


$$
\begin{gathered}
\frac{d^{2} e_{V}(t)}{d t^{2}}+2 \delta_{F} \omega_{n F} \frac{d e_{V}(t)}{d t}+\omega_{n F}^{2} e_{V}(t)=0 \\
e_{V}(t)=C \exp \left(-\delta_{F} \omega_{n F} t\right) \cdot \sin \left[\left(\omega_{n F} \sqrt{1-\delta_{F}^{2}}\right) t+\phi\right]
\end{gathered}
$$

where $\mathrm{C}$ is an arbitrary constant and $\phi$ is an arbitrary phase, which both depend on the initial conditions. From the previous equation is clear that $\lim _{t \rightarrow \infty} e_{V}(t)=0$ and therefore $V^{\prime}(t) \rightarrow V_{d}^{\prime}(t)$ as $t \rightarrow \infty$, so by choosing adequate values for $\omega_{n F}$ and $\delta_{F}$ the mobile piston can reach a prescribed behavior.

Next, the thermal subsystem must be analyzed. It should be recalled that the equation of thermal balance can be written in deviation variables according to Eqs (11) and (16). Additionally, we assume that the nonlinear function $f(T)$ of $\mathrm{Eq}(14)$ is chosen so that it verifies the following equation:

$$
\begin{gathered}
\frac{Q^{\prime}(t)+Q_{s}}{n c_{v}(t)}+\frac{K_{s} f(T)}{n c_{v}(t)}\left[\alpha\left[Q^{\prime}(t)+Q_{s}\right]-\beta T^{\prime}(t)\right]+\frac{\left[T^{\prime}(t)+T_{s}\right]}{n c_{v}(t)}\left(\frac{\partial P}{\partial T}\right)_{V} \frac{d V^{\prime}(t)}{d t}= \\
-K_{P T}\left[T^{\prime}(t)-T_{d}^{\prime}(t)+\frac{1}{\tau_{i T}} \int_{t_{a}}^{t}\left[T^{\prime}(\tau)-T_{d}^{\prime}(\tau)\right] d \tau\right]
\end{gathered}
$$

where $K_{P T}$ and $\tau_{i T}$ are the proportional and integral constants of a PI controller, whereas $t_{a}$ is an arbitrary time from which the new control law is applied. It should be noted that when this control law is substituted into Eq (11), by using the deviation variables of Eq (16) the following equations are obtained:

$$
\begin{aligned}
& \frac{d T^{\prime}(t)}{d t}=-K_{P T}\left[T^{\prime}(t)-T_{d}^{\prime}(t)+\frac{1}{\tau_{i T}} \int_{t_{a}}^{t}\left[T^{\prime}(\tau)-T_{d}^{\prime}(\tau)\right] d \tau\right]+\frac{d T_{d}^{\prime}(t)}{d t} \\
& e_{T}(t)=T^{\prime}(t)-T_{d}^{\prime}(t) \\
& \frac{d^{2} e_{T}(t)}{d t^{2}}+K_{p T} \frac{d e_{T}(t)}{d t}+\frac{K_{p T}}{\tau_{i T}} e_{T}(t)=0 \Rightarrow \frac{d^{2} e_{T}(t)}{d t^{2}}+2 \delta_{T} \omega_{n T} \frac{d e_{T}(t)}{d t}+\omega_{n T}^{2} e_{T}(t)=0
\end{aligned}
$$


It is clear that Eqs (74) and Eq (76) are similar, so by changing the control law at $t=t_{a}$ it will be possible to drive the motion of the mobile piston and the gas temperature to a prescribed state which is defined by the variables $V_{d}^{\prime}(t)$ and $T_{d}^{\prime}(t)$. This reasoning has been implemented by defining the respective desired values $P_{d}(t)$ and $T_{d}(t)$ for the gas pressure and temperature as follows:

$$
\left.\begin{array}{l}
P_{d}(t)=P_{0}+P_{a} \sin \omega_{t} t \\
T_{d}(t)=T_{0}+T_{a} \sin \omega_{t} t
\end{array}\right\}
$$

where $P_{0}, T_{0}, P_{a}, T_{a}$ are chosen to obtain a trajectory far away from the critical point, and $\omega_{t}$ is an angular frequency which defines the number cycles (together with the maximum simulation time). From the values of $P_{d}(t)$ and $T_{d}(t)$, the desired volume of the gas $\mathrm{V}_{\mathrm{d}}(\mathrm{t})$ is calculated through the Soave-Redlich-Kwong state equation given by Eq (12). Next, deviation variables are introduced and it is assumed that the control law is changed at $t=t_{a}=9.99 \mathrm{~s}$ with the following parameter values: $P_{0}=150 \mathrm{~atm}>P_{c r}=$ $33.94 \mathrm{~atm}, T_{0}=230 \mathrm{~K}>T_{c}=126.1 \mathrm{~K}, P_{a}=50 \mathrm{~atm}, T_{a}=50 \mathrm{~K}$ and $\omega_{t}=0.628 \mathrm{rad} / \mathrm{s}$. As it can be appreciated in figure 16, after a short transitory the gas pressure $P(t)$ and temperature $T(t)$ clearly tend to follow the desired values $P_{d}(t)$ and $T_{d}(t)$ respectively.

Finally, it should be noted that both the gas pressure and temperature oscillate chaotically for $t<t_{a}$. However, due to the effect of the PI control for the temperature defined in Eq (9), the amplitude of the temperature oscillations remains very small as it can be appreciated in Fig $12 \mathrm{c}$ ).

\section{Figure 16}

\section{Analysis of the numerical results}

In this section we shall corroborate the stability of the global control structure, and in addition we shall investigate the range of pressures and temperatures for which the numerical results obtained with the SRK and RK state equations can be regarded as physically meaningful. 
The control force on the mobile piston is defined in Eq (24). It should be noted that the purpose of this control force is to compensate the term $\left(S^{2} / m\right)\left[P^{\prime}(t)+P_{s}\right]$ due to the gas pressure, whereas the rest of the terms are introduced to obtain a homoclinic orbit so that the Melnikov method can be applied to deduce necessary conditions for chaotic behavior. Substituting the previous equation into the first Eq of (20) (which represents model of the mechanical subsystem) it is deduced that:

$$
\frac{d^{2} V^{\prime}(t)}{d t^{2}}=-\frac{K_{1}}{m}\left[V^{\prime}(t)+V_{s e}\right]-\frac{K_{2}}{m S^{2}}\left[V^{\prime}(t)+V_{s e}\right]^{3}-\left(f_{p}-1\right) \frac{b}{m} \frac{d V^{\prime}(t)}{d t}+\frac{K}{m S}\left[V^{\prime}(t)+V_{s e}\right]^{2}+\frac{S^{2}}{m} P_{1 s}
$$

From Eq (78) it is clear that the mechanical subsystem is not affected by the thermal one. However, the mechanical subsystem is always acting over the thermal one, since the partial derivative $(\partial P / \partial T)_{v}$ is always present in the equations of the thermal subsystem. For example, from the Soave-Redlich-Wong state equation given by Eq (12) $(\partial P / \partial T)_{v}$ has been calculated in Eq (68) where the partial derivative must be evaluated at each simulation time and consequently $\mathrm{x}_{1}(\mathrm{t})$ acts in the thermal system. Similar equations can be obtained for the Redlich-Wong state equation.

On the other hand, using deviation variables and taking into account the equations associated to the thermal subsystem in Eq (20) and Eqs (29), it is deduced that the matrix of the linear part of the complete system can be written as:

$$
A=\left[\begin{array}{cccc}
0 & 1 & 0 & 0 \\
-p_{s} & -\left(1-f_{p}\right) & 0 & 0 \\
0 & 0 & -\frac{\beta K_{s}}{n} & \frac{\alpha K_{s}}{n} \\
0 & 0 & -\left(\frac{K_{s} K_{P}}{\tau_{I}}-\frac{\beta K_{s}^{2} K_{p}}{n}\right) & -\frac{\alpha K_{s}^{2} K_{p}}{n}
\end{array}\right]
$$

where the effect of the decoupling between the mechanical and thermal subsystems can clearly be appreciated. The eigenvalues of matrix A are given by Eqs (21) and (33), and considering that the integral action $\tau_{\mathrm{i}}$ of the PI controller for the temperature has been 
chosen in accordance with Eqs (22) and (23), it is deduced that the eigenvalues of the thermal subsystem given by Eq (21) have negative real parts. On the other hand, taking into account the discussion of $\mathrm{Eq}$ (33) it is concluded that the eigenvalues of the mechanical subsystem have negative real parts for $f_{p}>1$ and $f_{p}<1$, and consequently A is a Hurwitz matrix. The case $f_{p}=1$ has been analyzed in subsection 3.5 and in the Appendix.

Next, we are going to study the results obtained with the compressibility diagram. Although our analysis shall focus on the chaotic regime, the results of this analysis can be easily extended to steady-state and oscillating behaviors. First we shall construct of the compressibility chart for the nitrogen by using the SRK state equation. The construction procedure is carried out through the following steps:

- The compressibility coefficient $v$ is defined as:

$$
v=z \frac{R T}{P}
$$

According to $\mathrm{Eq}$ (80), Eq (12) can be written as a function of the reduced pressure $P_{r}$ and the reduced temperature $T_{r}[10]$ as follows:

$$
\left.\begin{array}{c}
z^{3}-z^{2}+\left(A-B-B^{2}\right) z-A B=0 \\
A=0.42747 \bar{\alpha} \frac{P_{r}}{T_{r}^{2}} ; B=0.08664 \frac{P_{r}}{T_{r}}
\end{array}\right\}
$$

- Taking $T_{r}=1$, the cubic equation (81) has only one real root for each value of $P_{r}$ and thus the critical isotherm can be obtained.

- The same procedure of the two previous steps can be applied when $T_{r}>1$, and consequently the isotherms can be plotted in the zone of vapor at high temperature.

- The biphasic zone is less important for our purposes, since it is assumed that the range of pressures and temperatures lies outside this zone. Nevertheless, this zone has been plotted in Fig 16 by using the standard procedure [5], [28], [30]. 
Fig 17 depicts the simulation results obtained with the SRK and RK state equations when the system is in chaotic regime. It is interesting to remark that both state equations lead to a behavior that can be regarded as isotherm. In this sense, the results of the SRK state equation fit very well to the isotherm of $201.76 \mathrm{~K}$ (in accordance with the results shown in Fig 12), although the error for the RK state equation is slightly higher. In addition, it has been verified that the results shown in Fig 16 (especially in the region of vapor state) are almost coincident with the experimental compressibility charts published for the nitrogen [5], [31]. In summary, it can be affirmed that the results obtained for the system in chaotic regime are reasonably consistent for pressures and temperatures that are far away from the critical point.

Figure 17

In accordance with Fig 17, the previous analysis corroborates that the parameter values and the mathematical model are valid for high pressures and low temperatures above the critical point, i.e. when the pressure and gas temperature are sufficiently far away from the values $T_{c}=126.1 \mathrm{~K}$ and $P_{c}=33.94 \mathrm{~atm}$.

\section{Conclusions}

In this paper we have analyzed a system consisting of nitrogen (regarded as a real gas) inside of a cylinder with a mobile piston actuated by an external control force, a nonlinear spring and a viscous damper. Another thermal subsystem formed by two heating coils has been applied to supply heat to the gas so that the whole system can reach steady state, oscillating and chaotic behaviors.

To study the nitrogen behavior, two state equations have been analyzed: the Soave-Redlich-Kwong (SRK) and the Redlich-Kwong (RK) equations. One of the purposes of this work has been to investigate how to assign parameter values in the mechanical and thermal subsystems to obtain physically feasible results. Besides, it has been discussed which of the previous state equations (SRK and RK) gives better results in the presence of oscillating and chaotic behaviors. 
The mathematical model of the considered system is based on Newton laws and on the first principle of Thermodynamics. An external control force to compensate the high gas pressure has been applied on the mobile piston. Consequently, canceling the term associated to gas pressure, it has been achieved that the thermal subsystem does not act over the mechanical one, and thus only the mechanical subsystem can act over the thermal one. In addition, this control force allows an adequate choice of the nonlinear spring constants and the viscous damper coefficients to obtain physically admissible piston vibration frequencies.

The control force and parameter values of the mechanical subsystem have been chosen to obtain three equilibrium points for the piston position, showing that two of them can be stable focuses or nodes whereas the third one is an unstable saddle. As a consequence, it is shown that a family of homoclinic orbits can appear depending on the parameter values.

To verify the suitability of the parameter choice for the oscillation frequencies and amplitudes, it has been shown that the oscillating motion can be approximated through a successive approximation method based on the Laplace transform. The obtained results predict frequencies and amplitudes which are in very good agreement with the simulation results.

To obtain physically feasible simulation values, the specific heat at constant volume has been calculated through the SRK and RK state equations from accurate empirical data for the specific heat at constant pressure taking into account the correction due to the high gas pressure. The simulation results are consistent with the classical thermodynamic inequalities in steady-state, as well as in oscillating and chaotic regimes.

From the analysis of the thermal subsystem it has been shown that it is possible to obtain an approximate model for the gas temperature, whose analytical solution is very close to the simulated one. This additional aspect proves the consistency of the parameter values and mathematical model as well as of the designed thermal control system. 
The simulation results have shown that the thermodynamic equilibrium condition is exactly fulfilled with the SRK state equation, whereas with the RK state equation it is not exactly fulfilled. This discrepancy is probably due to the fact that the SRK and RK state equations have three and two adjustable parameters respectively. Nevertheless, both state equations provide similar values for the pressures, temperatures and gas molar volumes.

The Melnikov method to obtain necessary conditions for chaotic oscillations has been applied by using the homoclinic orbits, showing that it is possible to define a parameter zone where the system is potentially chaotic. Although there is no currently a definitive proof to elucidate when a dynamic system is chaotic, the sensitive dependence, Lyapunov exponents and power spectral density have been calculated to obtain reasonable indicators of chaos.

The simulation results show that all state variables have sensitive dependence, and the appearance of two positive Lyapunov exponents suggest an hyper-chaotic behavior. It has been corroborated that the sum of Lyapunov exponents is almost coincident with the mean value of the divergence of the vector field of the system, which can be regarded as one additional proof that the numerical simulations are reasonably correct. Similarly, the power spectral density is formed by multiple frequencies with decreasing intensity, which is a characteristic feature of chaotic systems.

In the last manuscript section, a discussion regarding the validity of the simulation results has been presented. The compressibility chart for the nitrogen has been calculated by using the SRK state equation, showing that the pressures, temperatures and compressibility coefficients are very close to the reported experimental data. The graphical representation of the data obtained for the chaotic regime shows that the simulation results are compatible with the compressibility chart for the SRK and RK state equations. Finally, it has been corroborated that the mathematical model and the parameter values are fully consistent if the pressures and temperatures are sufficiently far away from the critical point. 
As a concluding remark, this paper shows a framework in which nonlinear mechanics, classical thermodynamics, design of linear and nonlinear control systems, the application of approximation methods to study steady state and oscillating regime, the Melnikov theory and chaotic oscillations are presented from a unified viewpoint. The methodology of these investigations could be extended to more complicated situations, such as when the gas dynamics is inside the biphasic zone or when there is a mixture of different gases.

\section{Appendix}

In this appendix, the calculation of Eqs (51) and the third-order approximation of Eq (46) will be demonstrated. According to Eqs (48), the third-order approximation implies the calculation of the transform Laplace of $x_{1}(t) \cdot x_{2}(t)$ and $x_{1}^{2}(t) \cdot x_{2}(t)$. So taking into account $\mathrm{Eq}(50), \mathrm{Eq}(49)$ can be rewritten as:

$$
\begin{gathered}
x_{2}(t)=n_{1}+n_{2} \cos \omega_{1} t+n_{3} \cos 2 \omega_{1} t+n_{4} \cos 3 \omega_{1} t \\
n_{1}=-\frac{q_{s} A_{0}^{2}}{2 \omega_{1}^{2}} ; n_{2}=\frac{q_{s} A_{0}^{2}}{3 \omega_{1}^{2}}-\frac{r_{s} A_{0}^{3}}{32 \omega_{1}^{2}} ; n_{3}=\frac{q_{s} A_{0}^{2}}{6 \omega_{1}^{2}} ; n_{4}=\frac{r_{s} A_{0}^{3}}{32 \omega_{1}^{2}}
\end{gathered}
$$

From Eq (A1) we can deduce that:

$$
\begin{gathered}
\boldsymbol{L}\left[x_{1}(t) x_{2}(t)\right]=A_{0}\left[n_{1} G_{1}(s)+n_{2} G_{2}(s)+n_{3} G_{3}(s)+n_{4} G_{4}(s)\right] \\
G_{1}(s)=\frac{s}{s^{2}+4 \omega_{1}^{2}} ; G_{2}(s)=\frac{s^{2}+2 \omega_{1}^{2}}{s\left(s^{2}+4 \omega_{1}^{2}\right)} \\
G_{3}(s)=\frac{s\left(s^{2}+5 \omega_{1}^{2}\right)}{\left(s^{2}+\omega_{1}^{2}\right)\left(s^{2}+9 \omega_{1}^{2}\right)} ; G_{4}(s)=\frac{s\left(s^{2}+10 \omega_{1}^{2}\right)}{\left(s^{2}+16 \omega_{1}^{2}\right)\left(s^{2}+4 \omega_{1}^{2}\right)} \\
\boldsymbol{L}\left[x_{1}^{2}(t) x_{2}(t)\right]=A_{0}^{2}\left[n_{1} H_{1}(s)+n_{2} H_{2}(s)+n_{3} H_{3}(s)+n_{4} H_{4}(s)\right] \\
H_{1}(s)=\frac{s^{2}+2 \omega_{1}^{2}}{s\left(s^{2}+4 \omega_{1}^{2}\right)} ; H_{2}(s)=\frac{s\left(s^{2}+7 \omega_{1}^{2}\right)}{\left(s^{2}+\omega_{1}^{2}\right)\left(s^{2}+9 \omega_{1}^{2}\right)} \\
H_{3}(s)=\frac{s^{4}+14 \omega_{1}^{2} s^{2}+16 \omega_{1}^{4}}{s\left(s^{2}+4 \omega_{1}^{2}\right)\left(s^{2}+16 \omega_{1}^{2}\right)} ; H_{4}(s)=\frac{\left.s_{1}^{2} s^{2}+71 \omega_{1}^{4}\right)}{\left(s^{2}+\omega_{1}^{2}\right)\left(s^{2}+9 \omega_{1}^{2}\right)\left(s^{2}+25 \omega_{1}^{2}\right)}
\end{gathered}
$$


On the other hand, the third equation of (48) allows to express the Laplace transform of $x_{3}(t)$ as:

$$
\begin{gathered}
X_{3}(s)=\frac{-C_{3} X_{1}(s)}{s^{2}+\omega_{1}^{2}}+X_{31}(s)+X_{32}(s)+X_{33}(s) \\
X_{30}(s)=\frac{-C_{3} X_{1}(s)}{s^{2}+\omega_{1}^{2}} ; X_{31}(s)=\frac{-C_{2} X_{2}(s)}{s^{2}+\omega_{1}^{2}} \\
X_{32}(s)=\frac{-2 q_{s} \boldsymbol{L}\left[x_{1}(t) x_{2}(t)\right]}{s^{2}+\omega_{1}^{2}} ; X_{33}(s)=\frac{-3 r_{s} \boldsymbol{L}\left[x_{1}^{2}(t) x_{2}(t)\right]}{s^{2}+\omega_{1}^{2}}
\end{gathered}
$$

where:

$$
X_{1}(s)=\frac{s A_{0}}{\left(s^{2}+\omega_{1}^{2}\right)} \Rightarrow x_{1}(t)=A_{0} \cos \omega_{1} t
$$

According to Eqs (A2)-(A5) it is possible to obtain the inverse Laplace transform of the variables indicated in Eq (A4), which in turn allow to obtain the thirdorder approximation $x_{3}(t)$. For this purpose, the results of the intermediate calculations are the following ones:

$$
\begin{gathered}
x_{30}(t)=-\frac{C_{3} A_{0}}{2 \omega_{1}} t \sin \omega_{1} t \\
x_{31}(t)=C_{2}^{2} A_{0}\left(\frac{1}{8 \omega_{1}^{2}} t \sin \omega_{1} t-\frac{1}{8 \omega_{1}^{2}} t^{2} \cos \omega_{1} t\right)+ \\
+C_{2} q_{s} A_{0}^{2}\left(\frac{-1}{6 \omega_{1}^{3}} t \sin \omega_{1} t+\frac{1}{18 \omega_{1}^{4}} \cos 2 \omega_{1} t-\frac{5}{9 \omega_{1}^{4}} \cos \omega_{1} t+\frac{1}{2 \omega_{1}^{4}}\right)+ \\
+C_{2} r_{s} A_{0}^{3}\left(\frac{7}{64 \omega_{1}^{3}} t \sin \omega_{1} t-\frac{3}{32 \omega_{1}^{2}} t^{2} \cos \omega_{1} t+\frac{1}{256 \omega_{1}^{4}} \cos 3 \omega_{1} t-\frac{1}{256 \omega_{1}^{4}} \cos \omega_{1} t\right)
\end{gathered}
$$

It should be noted that taking into account the value of $C_{2}$ given in Eq (50), the secular term $t^{2} \cos \omega_{1} t$ disappears in Eq (A7). In addition, it can be easily checked that $x_{31}(0)=0$, which is an indirect proof of the correctness of the previous calculations. Similarly, for $x_{32}(t)$ and $x_{33}(t)$ it is deduced that: 


$$
\begin{gathered}
x_{32}(t)=-2 q_{s} A_{0} n_{1} \frac{1}{2 \omega_{1}} t \sin \omega_{1} t-2 q_{s} A_{0} n_{2}\left(-\frac{1}{6 \omega_{1}^{2}} \cos 2 \omega_{1} t-\frac{1}{3 \omega_{1}^{2}} \cos \omega_{1} t+\frac{1}{2 \omega_{1}^{2}}\right)+ \\
-2 q_{s} A_{0} n_{3}\left(\frac{1}{4 \omega_{1}} t \sin \omega_{1} t-\frac{1}{16 \omega_{1}^{2}} \cos 3 \omega_{1} t+\frac{5}{16 \omega_{1}^{2}} \cos \omega_{1} t\right)+ \\
-2 q_{s} A_{0} n_{4}\left(-\frac{2}{60 \omega_{1}^{2}} \cos 4 \omega_{1} t-\frac{2}{12 \omega_{1}^{2}} \cos 2 \omega_{1} t-\frac{1}{5 \omega_{1}^{2}} \cos \omega_{1} t\right) \\
x_{33}(t)=-3 r_{s} A_{0} n_{1}\left(-\frac{1}{6 \omega_{1}^{2}} \cos 2 \omega_{1} t-\frac{1}{3 \omega_{1}^{2}} \cos \omega_{1} t+\frac{1}{2 \omega_{1}^{2}}\right)- \\
-3 r_{s} A_{0}^{2} n_{2}\left(\frac{3}{8 \omega_{1}} t \sin \omega_{1} t-\frac{1}{32 \omega_{1}^{2}} \cos 3 \omega_{1} t+\frac{1}{32 \omega_{1}^{2}} \cos \omega_{1} t\right) \\
-3 r_{s} A_{0}^{2} n_{3}\left(-\frac{2}{12 \omega_{1}^{2}} \cos 4 \omega_{1} t-\frac{2}{12 \omega_{1}^{2}} \cos 2 \omega_{1} t-\frac{1}{15 \omega_{1}^{2}} \cos \omega_{1} t+\frac{1}{4 \omega_{1}^{2}}\right)- \\
-3 r_{s} A_{0}^{2} n_{4}\left(\frac{1}{8 \omega_{1}} t \sin \omega_{1} t-\frac{2}{32 \omega_{1}^{2}} \cos 3 \omega_{1} t-\frac{2}{192 \omega_{1}^{2}} \cos 5 \omega_{1} t+\frac{7}{96 \omega_{1}^{2}} \cos \omega_{1} t\right)
\end{gathered}
$$

Like in the calculation of $x_{31}(0)$, from Eqs (A8) and (A9) it can be verified that $x_{32}(0)=x_{33}(0)=0$. The last step is to calculate $C_{3}$ and the frequency $\omega_{1}$ in the thirdorder approximation given by Eq (51). To do this end, we make zero the sum of secular terms in Eqs (A6)-(A9) by considering $C_{3}=-C / \omega_{1}^{2}$, where $C$ is given in Eq (51). Finally, from Eq (47) it follows that:

$$
\omega^{2}=\omega_{1}^{2}-\frac{3}{4} r_{s} A_{0}^{2}-\frac{C}{\omega_{1}^{2}}
$$

Eq (A10) allows to obtain the value of $\omega_{1}$ in $\mathrm{Eq}(51)$. 


\section{References}

[1] L. D. Landau, E. M. Lifshitz, Statistical Physics, third ed., Part 1: Volume 5, Elsevier Butterwort-Heinemann, Reprinted 2005.

[2] G. N. Hatsopoulos, J. H. Keenan, Principles of General Thermodynamics, John Wiley \& Sons, New York, 1965

[3] E. A. Guggenhein, Thermodynamics and Advanced Treatment for Chemists and Physicists, Elsevier Science Publishers, Amsterdam, 1967.

[4] D. E. Winterbone, Advanced Thermodynamics for Engineers, John Wiley \& Sons, London, 1997.

[5] Kalyan Annamalai, Ishwar K. Puri, Advanced Thermodynamics Engineering, CRC Press, Boca Raton, Florida, 2002.

[6] D. Q. Kern, Process Heat Transfer, McGraw-Hill, New York, 1974.

[7] E. E. Ludwig, Applied Process Design for Chemical and Petrochemicals Plants, Vol. 3, Third Ed., Gulf Professional Publishing, Boston, 1994.

[8] O. Redlich, J. N. S. Kwong, On the Thermodinamics of Solutions. V: An Equation of State. Fugacities of Gaseous Solutions, Symposium on Thermodynamics and Molecular Structure of Solutions, 114th Meeting of the American Chemical Society, Portland, Oregon, September 13 and 14, 1948.

[9] G. Soave, Equilibrium constants from a modified Redlich-Kwong equation of state, Chem. Eng. Science, 27 (1972) 1197-1203.

[10] G. Soave, Improvement of the Van Der Waals equation of state, Chem. Eng. Science, 39 (1984) 357-369.

[11] Y. S. Wei, R.J. Sadus, Equations of State for the Calculation of Fluid-Phase Equilibria, AIChE Journal, 46 (2000) 160-196.

[12] Ding-Yu Peng and Donald B. Robinson, A New Two-Constant Equation of State, Ind. Eng. Chem., Fundam., 15, 1, (1976) 59-64.

[13] J. R. Elliot, T. E. Daubert, Revised Procedures for Phase Equilibrium Calculations with the Soave Equation of State, Ind. Eng. Chem. Process Des. Dev. 24 (1985) 743-753.

[14] Paul W. Belanger, Christopher D. Singley, Michael J. Misovich, Coexistence curve predicted by the soave-Redlich-Kwong equation in the critical limit, Chem. Eng. Science, 49 (1994) 3787-3789.

[15] K. Ogata, Modern Control Engineering, Prentice-Hall, New York (2000)

[16] P. Albertos, A. Sala, A, Multivariable Control Systems, Springer, London 2004.

[17] Manuel F. Perez-Polo, Manuel Perez-Molina, Increasing the reactant conversion through induced oscillations in a continuous stirred tank reactor by using PI control, Journal Process Control, 23 (2013) 778-792.

[18] A. Isidori, Nonlinear Control Nonlinear control systems, third ed. London, Springer, 2003.

[19] Manuel Perez-Molina, Manuel F. Perez-Polo, Fold-Hopf bifurcation, steady state, self-oscillating and chaotic behavior in an electromechanical transducer with nonlinear control, Commun. Nonlinear Sci. Numer. Simulat., 17 (2012) 129-152.

[20] Manuel F. Perez-Polo, Manuel Perez-Molina, Self-oscillating chaotic behavior and induced oscillations of a continuous stirred tank reactor with nonlinear control, Chem. Eng. J. 191 (2012) 512-527.

[21] A. Wagemakers, S. Zambrano, M. A. Sanjuán, Partial control of transient chaos in electronic circuits, International Journal Bifurcations and Chaos, 22 (2012) 1250032 . 
[22] J. Guckenheimer, P. Holmes, Nonlinear Oscillations, Dynamical Systems and Bifurcations of Vector Fields, Springer, New York 1983.

[23] S. Wiggins, Introduction to Applied Nonlinear Dynamical Systems and Chaos, second ed., Springer, New York, 2000.

[24] Miguel A.F. Sanjuán. Remarks on Transitions Order-chaos Induced by the Shape of the Periodic Excitation in a Parametric Pendulum. Chaos Solitons \& Fractals, 7, 3 (1996) 435-440.

[25] L.P. Shilnikov, A.L. Shilnikov, D.V. Turaev, L.O. Chua, Methods of Qualitative Theory in Nonlinear Dynamics. Part II, World Scientific, New Jersey, 2001.

[26] M. Siewe Siewe, C. Tchawoua, P. Woafo, Melnikov chaos in a periodically driven Rayleigh-Duffing oscillator, Mechanics Research Communications, 37 (2010) 363-368.

[27] Manuel F. Pérez-Polo, Manuel Pérez-Molina, Steady-state self oscillations and chaotic behavior of a controlled electromechanical device by using the first Lyapunov value and the Melnikov theory, Journal of Sound and Vibration, 333 (2014) 1163-1181.

[28] M. J. Moran, H. N. Shapiro, Fundamentals of Engineering Thermodynamics, fourth ed., John Wiley \& Sons, New York, 2004.

[29] Carl L. Yaws. Chemical Properties Handbook, McGraw-Hill, New York. 1999.

[30] O. A. Hougen and K. M. Watson, R. A. Ragatz, Industrial Chemical Calculations, Vol. II Thermodynamics, second ed., John Wiley \& Sons, New York, 1954.

[31] F. F. Huang, Engineering Thermodynamics. Fundamentals and Applications, MacMillan Publishing, New York, 1976.

[32] A.J. Lichtenberg, M.A. Lieberman, Regular and chaotic dynamics, second ed., Springer-Verlag, New York, 1992.

[33] Amir Fayazi, Milad Arabloo, Amir H. Mohammadi, Efficient estimation of natural gas compressibility factor using a rigorous method, Journal of Natural Gas Science and Engineering, 16 (2014) 8-17.

[34] G. Benettin, L. Galgani, A. Giorgilly, J.M. Strelcyn, Lyapunov characteristic exponents for smooth dynamical systems and for Hamiltonian systems: a method for computing all of them, part I: theory, Meccanica 15 (1980) 9-20.

[35] G. Benettin, L. Galgani, A. Giorgilly, J.M. Strelcyn, Lyapunov characteristic exponents for smooth dynamical systems and for Hamiltonian systems: a method for computing all of them, part II: numerical applications, Meccanica 15 (1980) 21-30.

[36] M.F.P. Polo, P. Albertos, Nonisothermal Stirred- Tank Reactor with Irreversible Exothermic Reaction A $\rightarrow$ B: Nonlinear Phenomena in: H. O. Méndez-Acosta, R. Femat, V. Gónzalez -Álvarez (Eds), Lecture Notes in Control and Information Sciences, $n^{\circ} 361$, Springer-Verlag, Berlin, Heilderberg, 2007, pp. 276-279. 
TABLE 1 PARAMETER VALUES

\begin{tabular}{|c|c|c|}
\hline Variable & Description & Value \\
\hline $\mathrm{L}$ & Length of the cylinder (m) & 1 \\
\hline$d_{c}$ & Diameter of the cylinder $(\mathrm{m})$ & 0.1 \\
\hline S & Cross sectional area of the cylinder $\left(\mathrm{m}^{2}\right)$ & $7.8540 \cdot 10^{-3}$ \\
\hline$d_{1}$ & Required distance for the heating coils (m) & 0.1 \\
\hline $\mathrm{V}_{\mathrm{s}}$ & Gas volume $\left(\mathrm{m}^{3}\right)$ & $6 \cdot 10^{-3}$ \\
\hline $\mathrm{V}_{\mathrm{se}}$ & Effective volume $\left(\mathrm{m}^{3}\right)$ & $5.6 \cdot 10^{-3}$ \\
\hline $\mathrm{V}_{\mathrm{c}}$ & Volume of the heating coils $\left(\mathrm{m}^{3}\right)$ & $9.8696 \cdot 10^{-5}$ \\
\hline$V_{\text {sd }}$ & Volume of the spring and damper $\left(\mathrm{m}^{3}\right)$ & $3.4240 \cdot 10^{-4}$ \\
\hline $\mathrm{c}$ & Piston width (m) & 0.02 \\
\hline$d_{c}$ & Internal diameter of the heating coils (m) & $5 \cdot 10^{-3}$ \\
\hline $\mathrm{D}_{\mathrm{c}}$ & External diameter of the heating coils (m) & 0.04 \\
\hline $\mathrm{N}$ & Number of turns of the heating coils & 20 \\
\hline $\mathrm{v}_{\mathrm{f}}$ & Fluid velocity within the heating coils $(\mathrm{m} / \mathrm{s})$ & $9.1440 \cdot 10^{-1}$ \\
\hline $\mathrm{h}$ & Heat transmission coefficient $\left(\mathrm{kJ} / \mathrm{s} \cdot \mathrm{m}^{2}\right)$ & 341.18 \\
\hline $\mathrm{K}_{\mathrm{s}}$ & Constant of the heating coils $\left(\mathrm{J} / \mathrm{m}^{3}\right)$ & $7.5020 \cdot 10^{8}$ \\
\hline$\alpha$ & Constant of nonlinear control law $\left(\mathrm{m}^{3} \cdot\right.$ mole. $\left.\mathrm{K} / \mathrm{J}^{2}\right)$ & $9.7262 \cdot 10^{-11}$ \\
\hline$\beta$ & Constant of nonlinear control law (mole. $\left.\mathrm{m}^{3} / \mathrm{J} . \mathrm{s}\right)$ & $1.333 \cdot 10^{-10}$ \\
\hline $\mathrm{K}_{\mathrm{p}}$ & Proportional constant of the PI controller $\left(\mathrm{m}^{3} / \mathrm{s} . \mathrm{K}\right)$ & $1.8268 \times 10^{-9}$ \\
\hline$\tau_{\mathrm{i}}$ & Reset time of the PI controller (s) & 0.1576 \\
\hline$f_{c}$ & Coefficient to calculate $\tau_{\mathrm{i}}$ & 0.001 \\
\hline $\mathrm{m}$ & Mass of the piston $(\mathrm{kg})$ & 1.1938 \\
\hline $\mathrm{K}_{1}$ & Linear constant of the nonlinear spring $(\mathrm{N} / \mathrm{m})$ & 311.64 \\
\hline $\mathrm{K}_{2}$ & Nonlinear constant of the nonlinear spring $\left(\mathrm{N} / \mathrm{m}^{3}\right)$ & 449.84 \\
\hline K & Constant of the control force $\left(\mathrm{N} / \mathrm{m}^{2}\right)$ & 680.14 \\
\hline$P_{1 s}$ & Pressure of the control force $\left(\mathrm{N} / \mathrm{m}^{2}\right)$ & 5286.63 \\
\hline $\mathrm{b}$ & Viscous damping $(\mathrm{N} / \mathrm{m} / \mathrm{s})$ & 1 \\
\hline $\mathrm{n}$ & Number of gas moles & 15.757 \\
\hline $\mathrm{T}_{\mathrm{s}}$ & Set point temperature $(\mathrm{K})$ & 200 \\
\hline $\mathrm{P}_{\mathrm{s}}$ & Initial pressure of the gas (atm) & 40 \\
\hline $\mathrm{Q}_{\mathrm{s}}$ & Steady-state heat flow in the heating coils $(\mathrm{J} / \mathrm{s})$ & $6.7346 \cdot 10^{3}$ \\
\hline$A_{p}$ & Amplitude of the external disturbance $\left(\mathrm{m}^{3} / \mathrm{s}^{2}\right)$ & 0.02 \\
\hline$\omega_{\mathrm{p}}$ & Frequency of the external disturbance $(\mathrm{rad} / \mathrm{s})$ & 5.2 \\
\hline
\end{tabular}




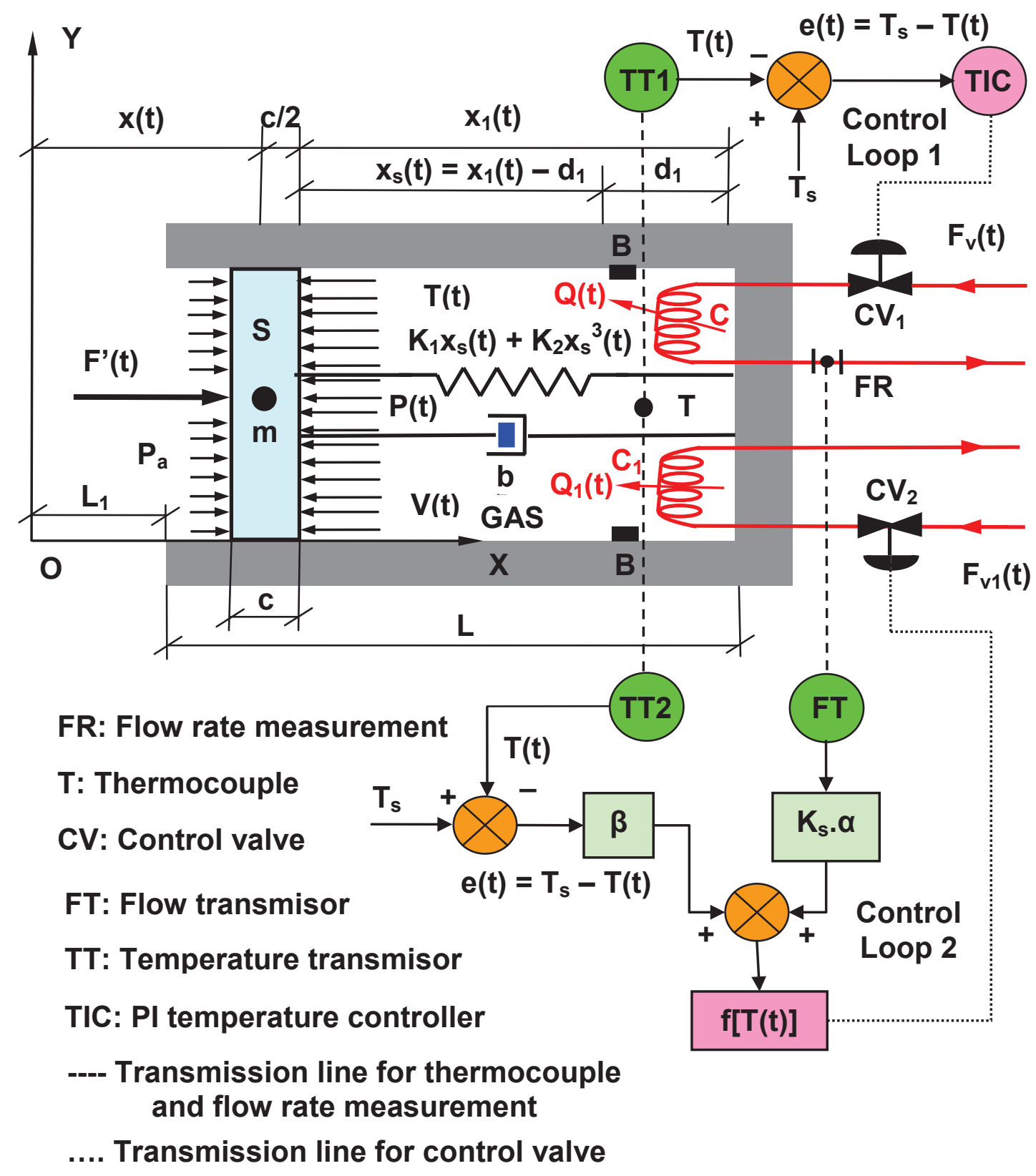

Figure 1 


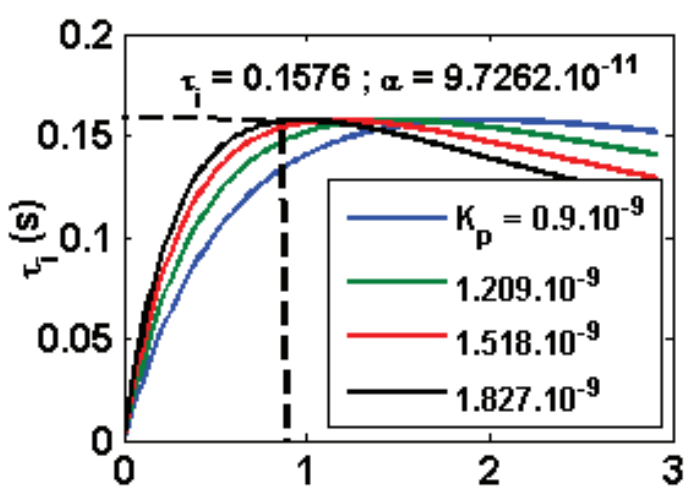

a) $\alpha\left(\mathrm{m}^{3} \cdot \mathrm{mol} \cdot \mathrm{KIJ} \mathrm{J}^{2}\right) \times 10^{-10}$

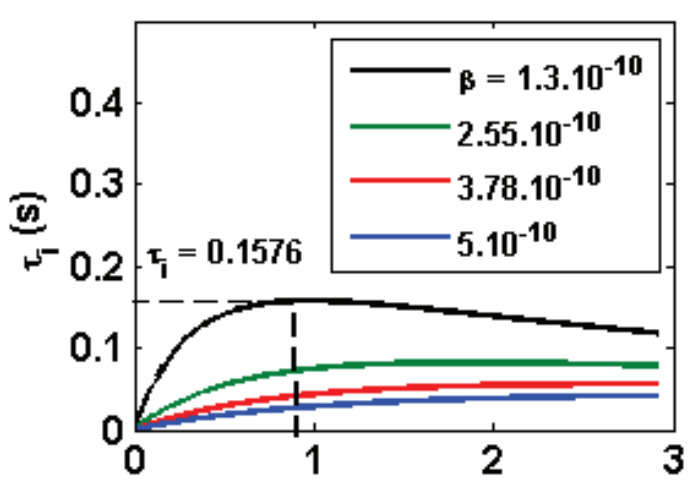

b) $\alpha\left(\mathrm{m}^{3} \cdot \mathrm{mol} \cdot \mathrm{KI} \mathrm{J}^{2}\right) \times 10^{-10}$

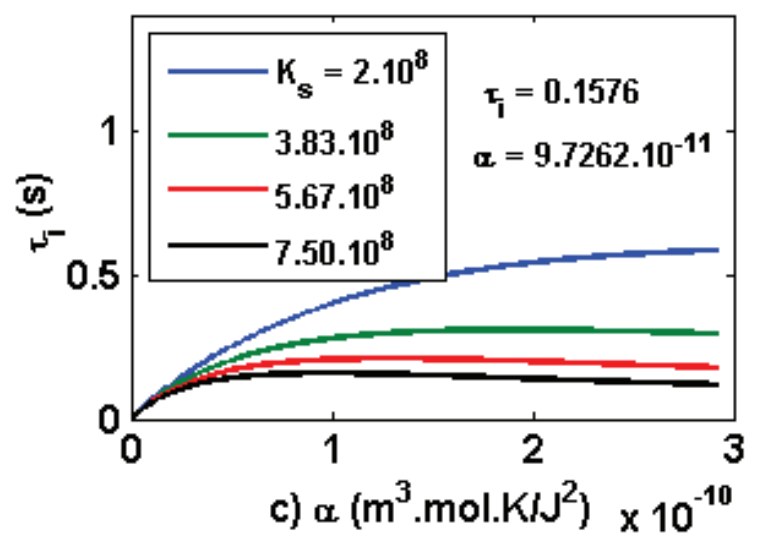

Figure 2 

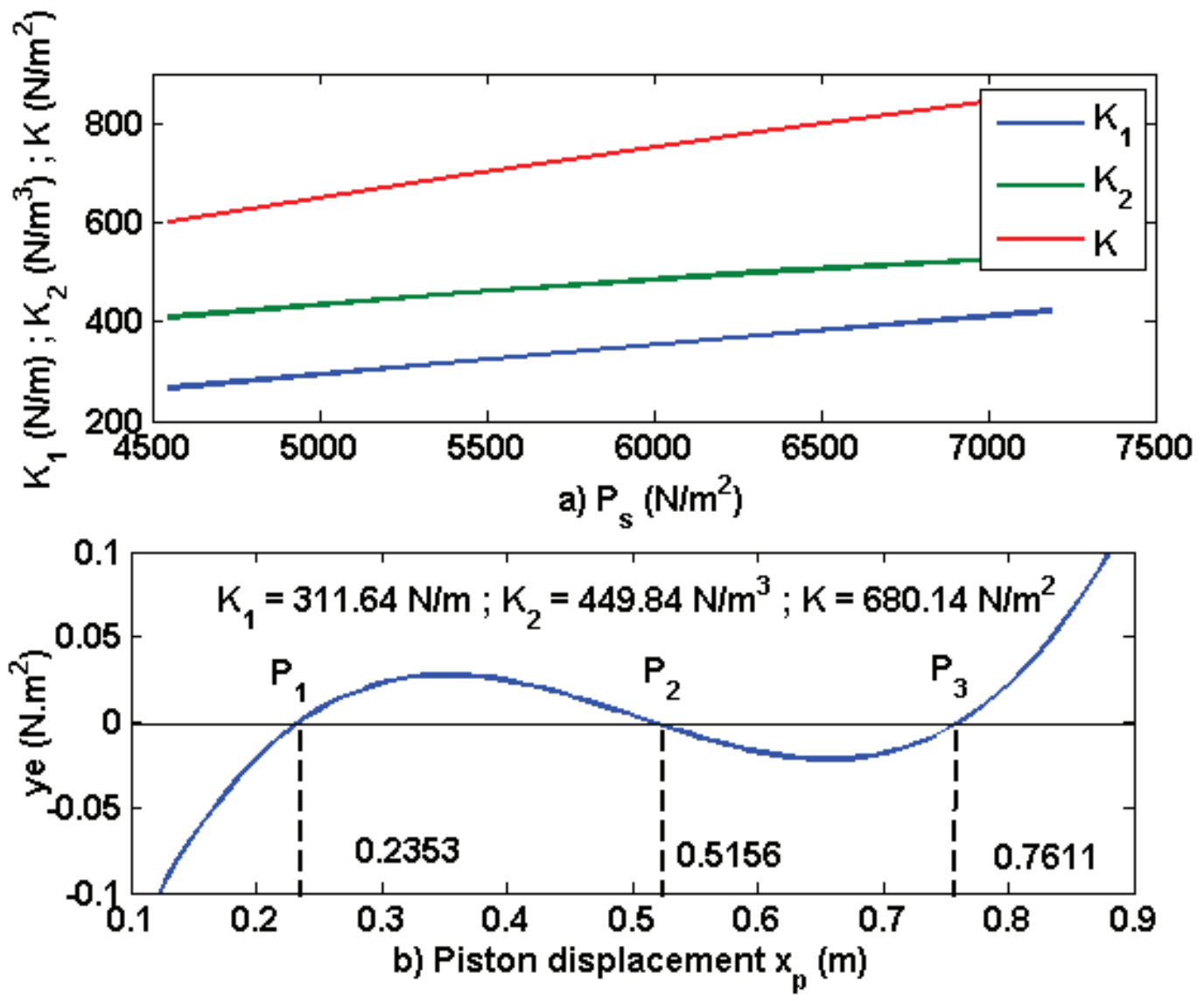

Figure 3 


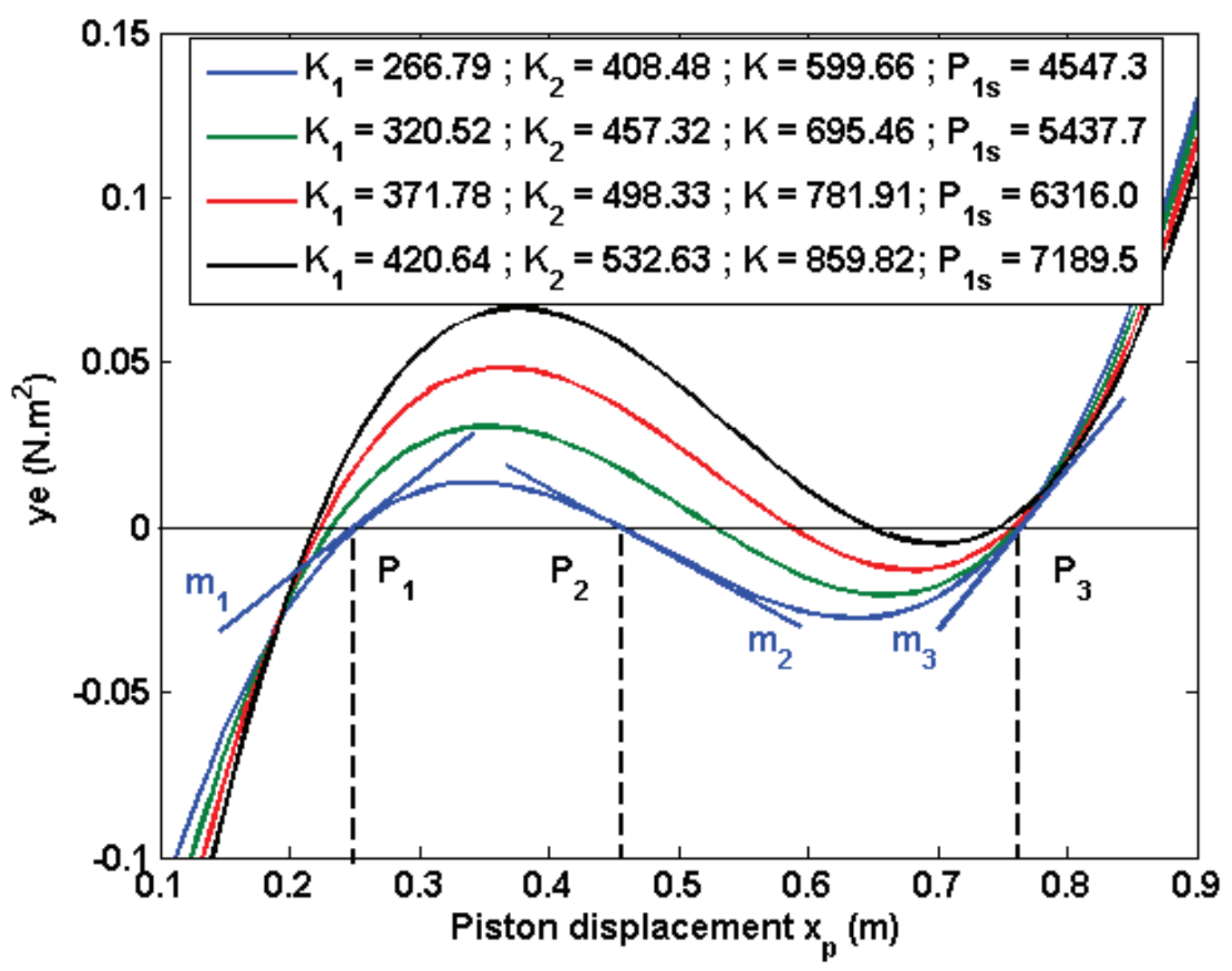

Figure 4 


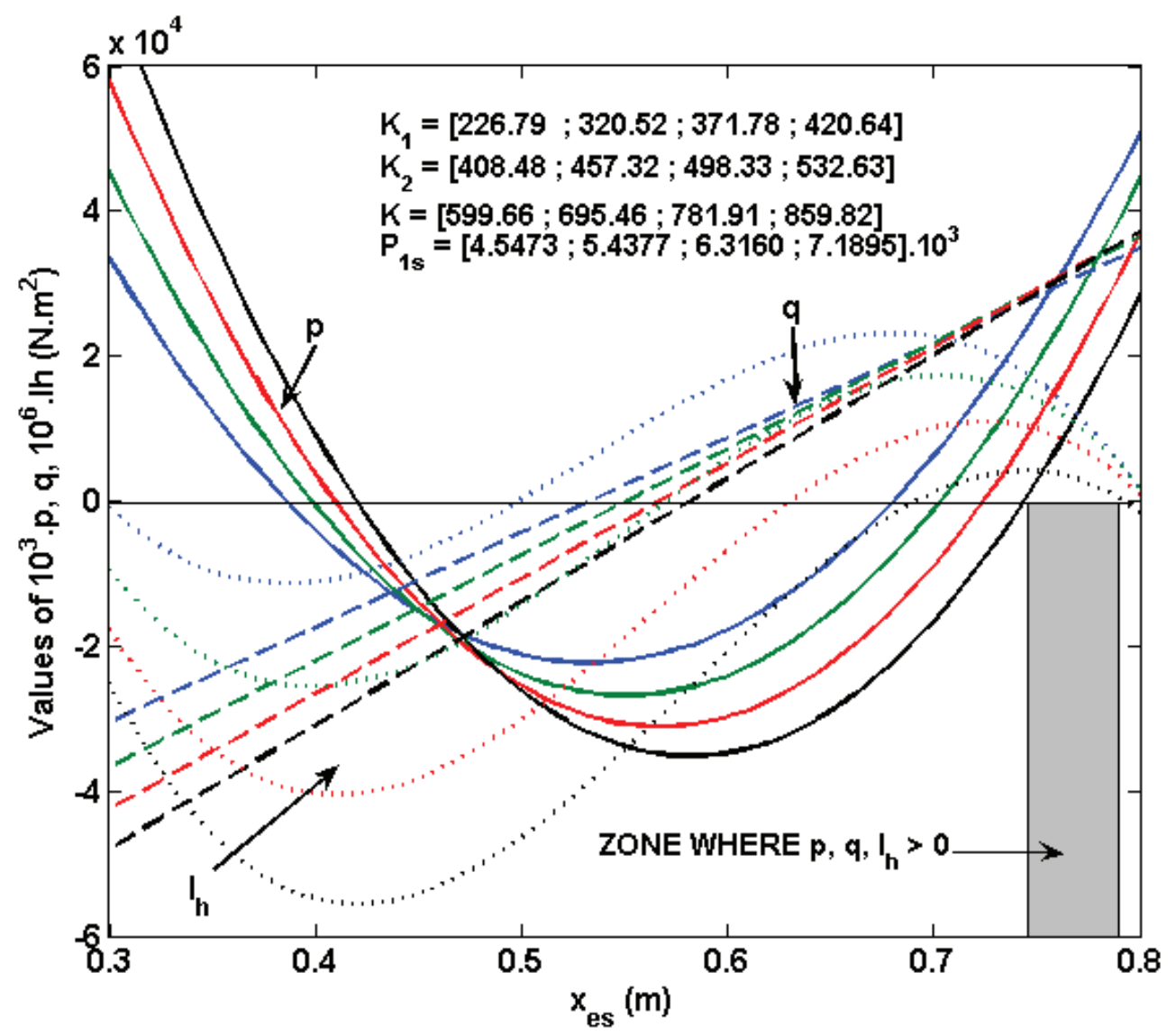

Figure 5 


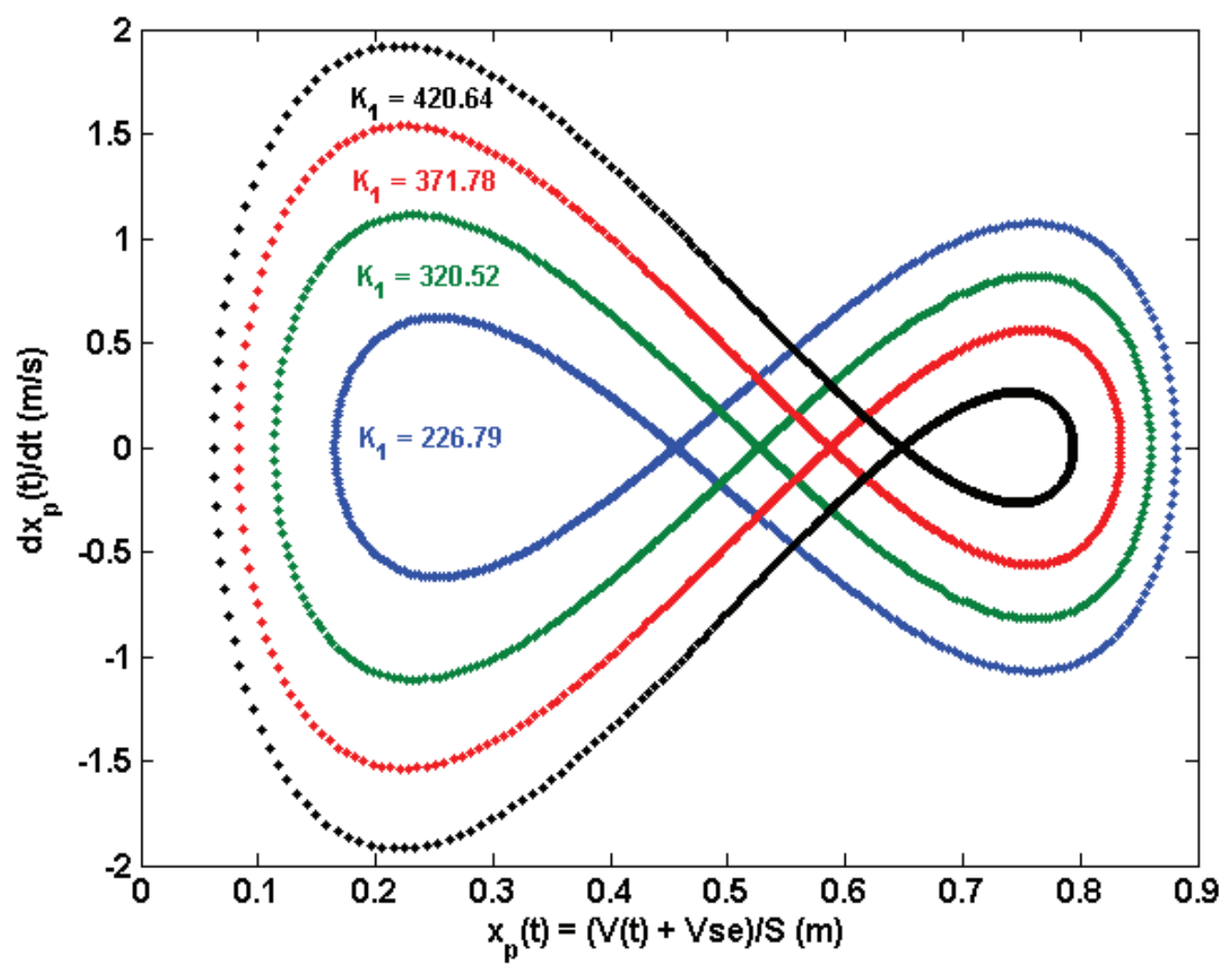

Figure 6 

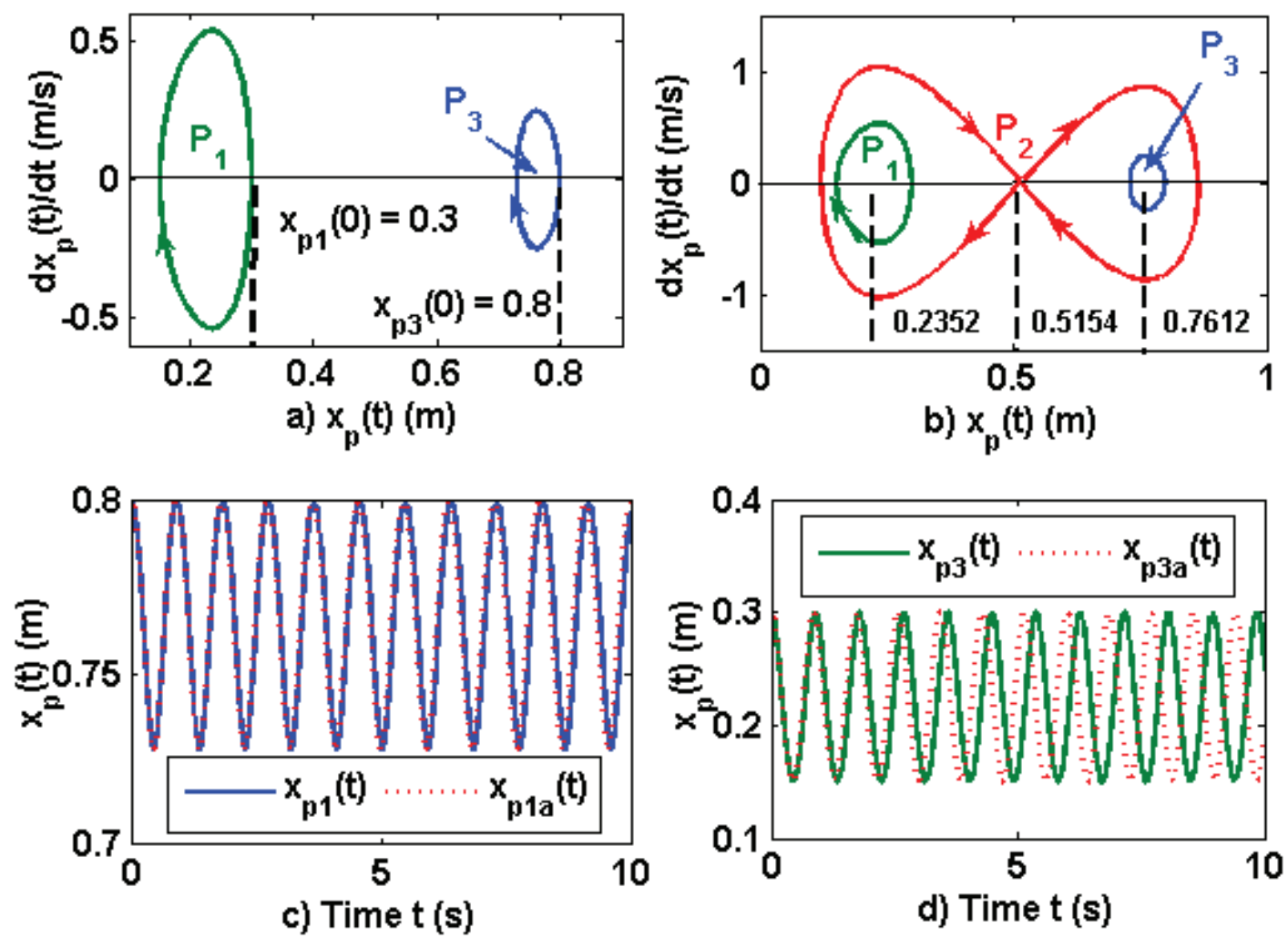

Figure 7 


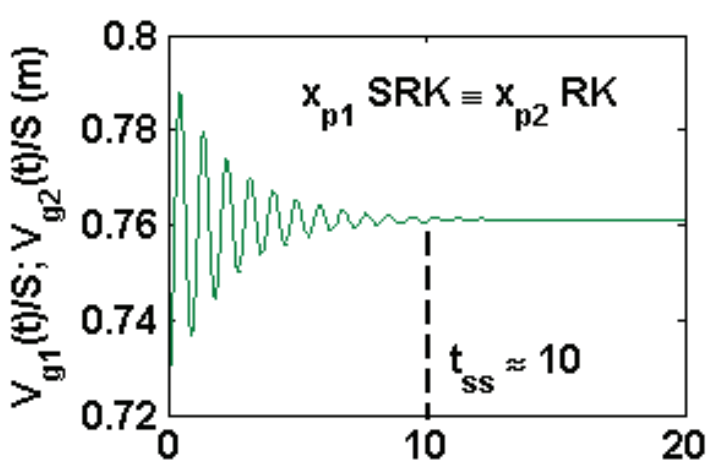

a) Time t (s)

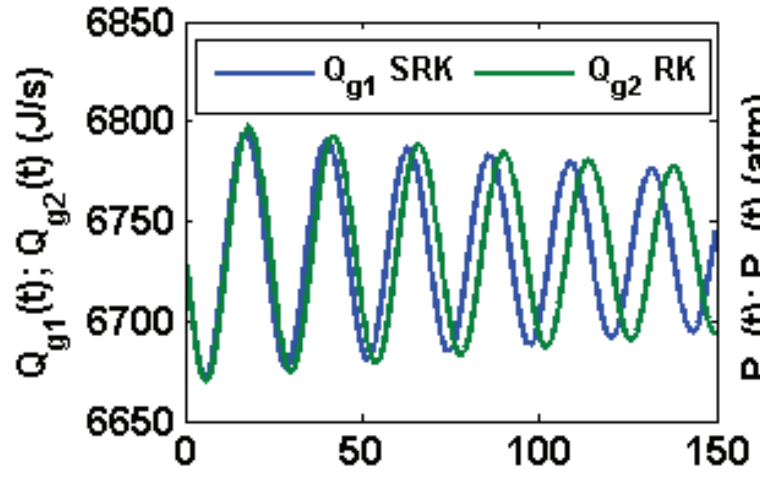

c) Time $t$ (s)

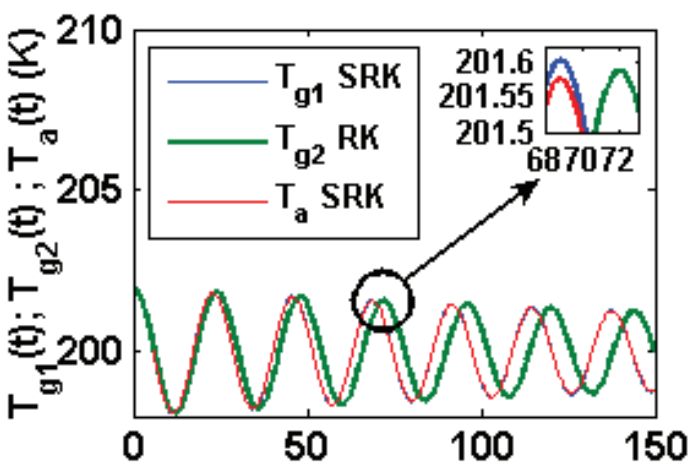

b) Time t (s)

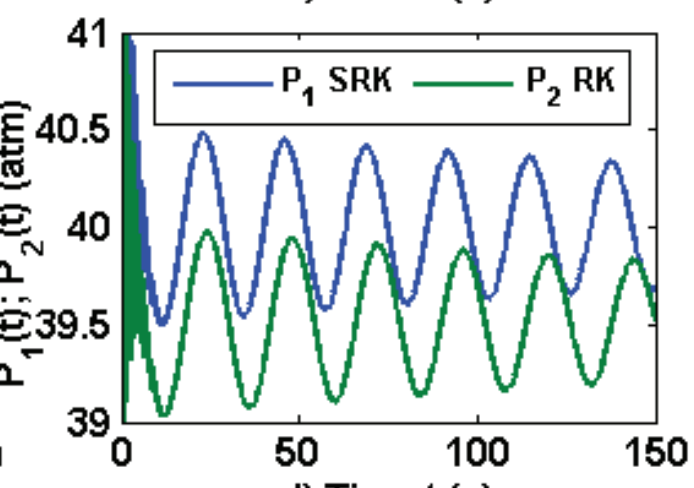

d) Time t (s)

Figure 8 


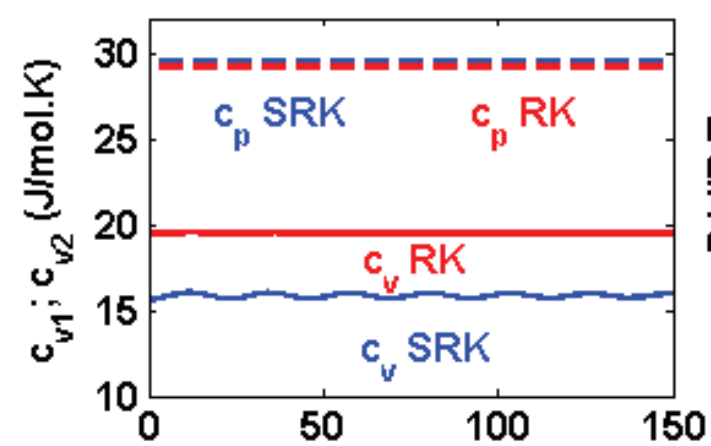

a) Time t (s)

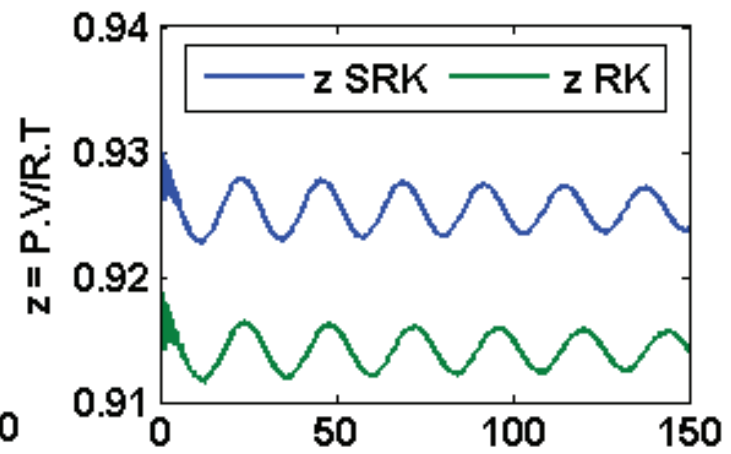

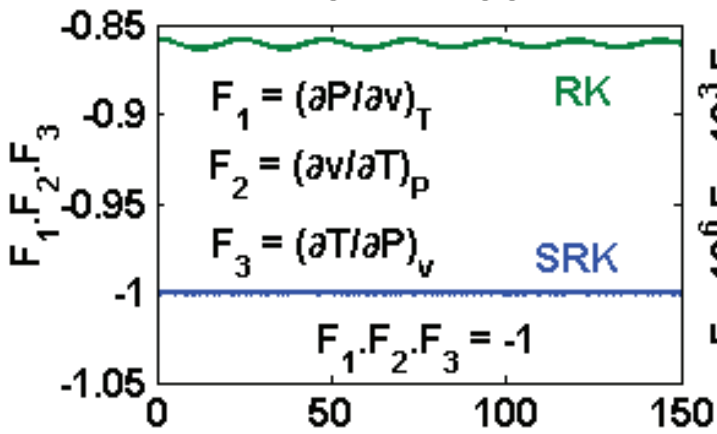

c) Time t (s) b) Time $t$ (s)

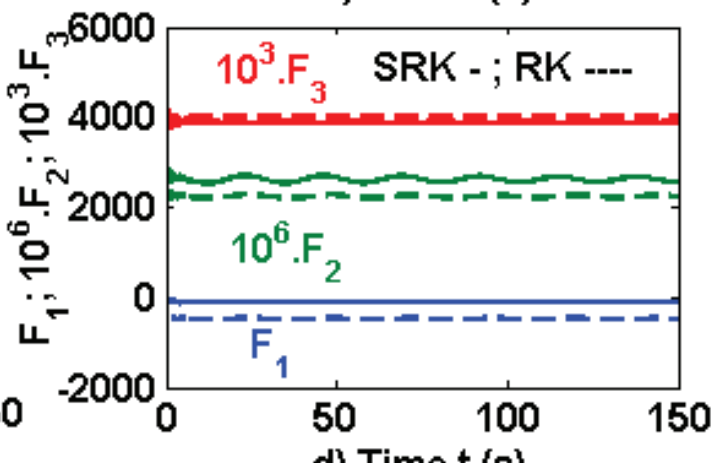

Figure 9 


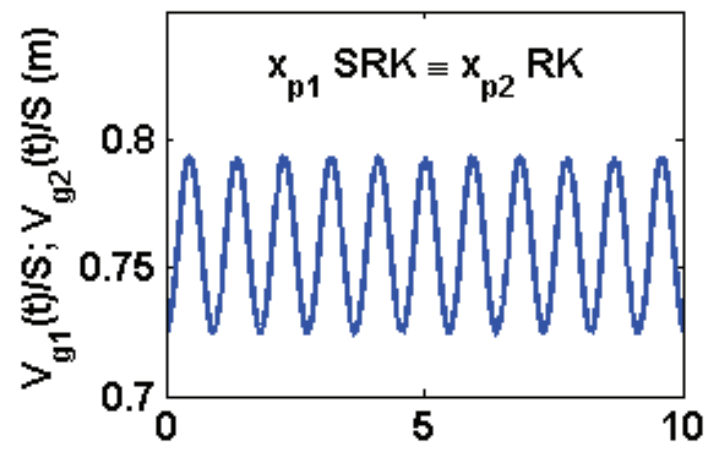

a) Time t (s)

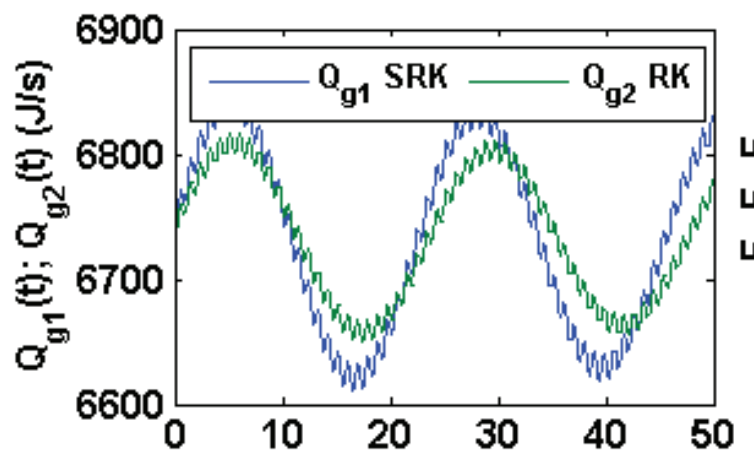

c) Time t (s)

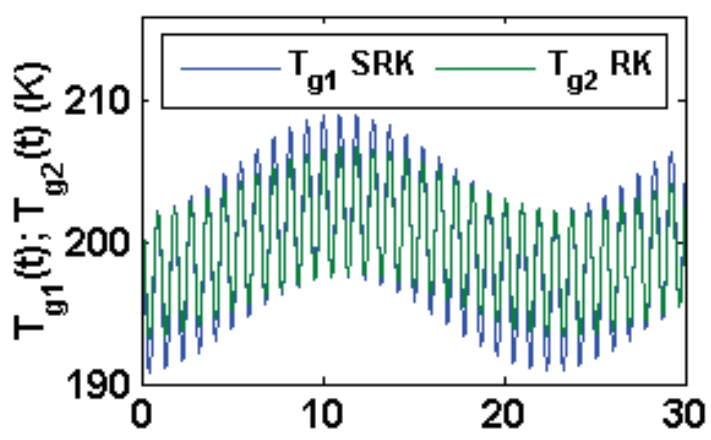

b) Time $t$ (s)

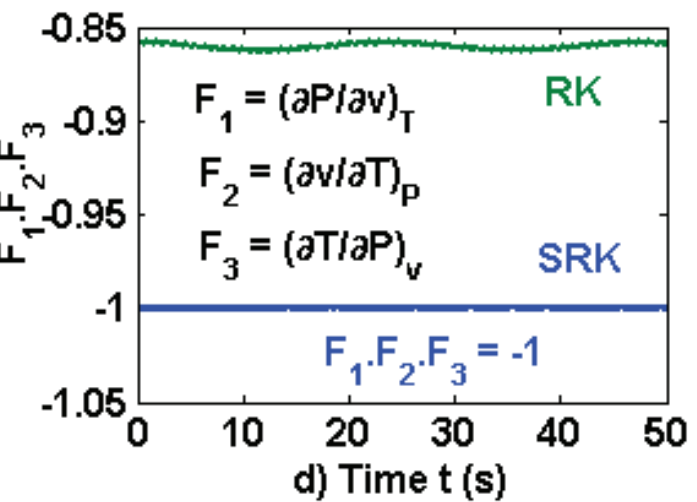

Figure 10 

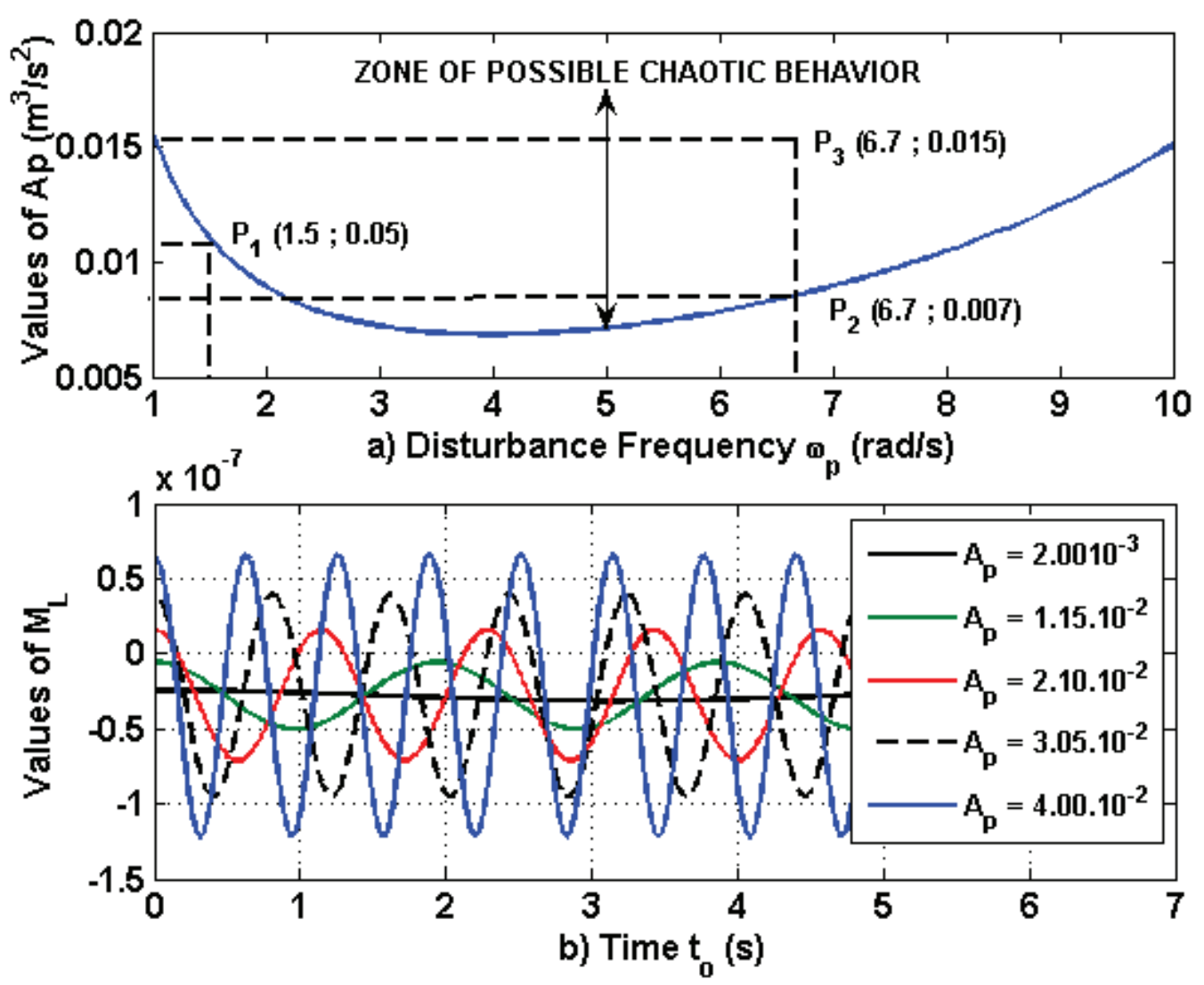

Figure 11 

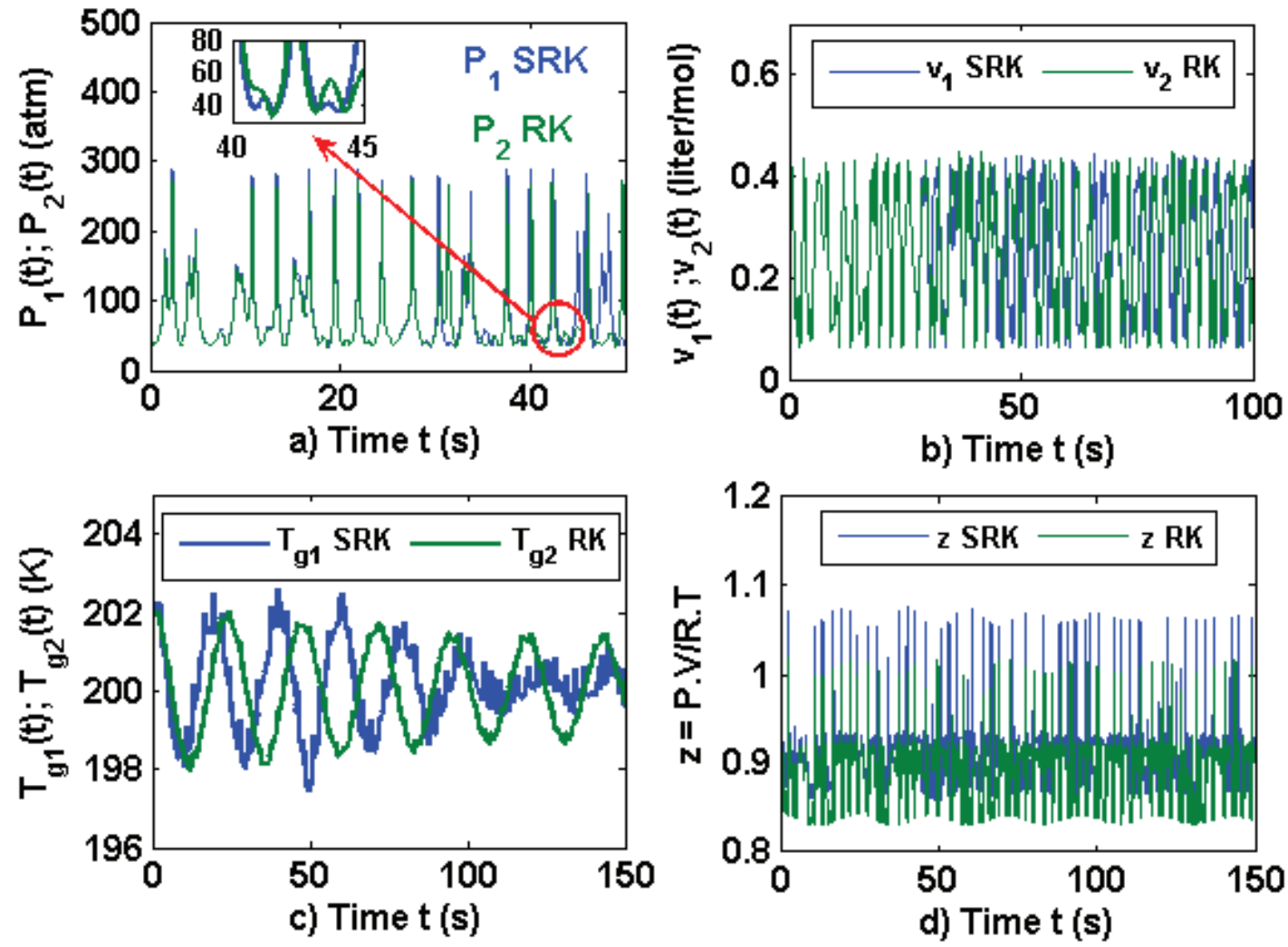

Figure 12 


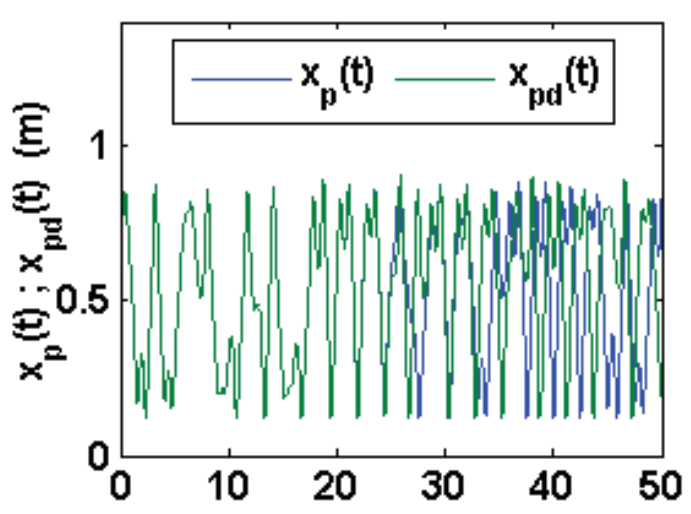

a) Time $t$ (s)

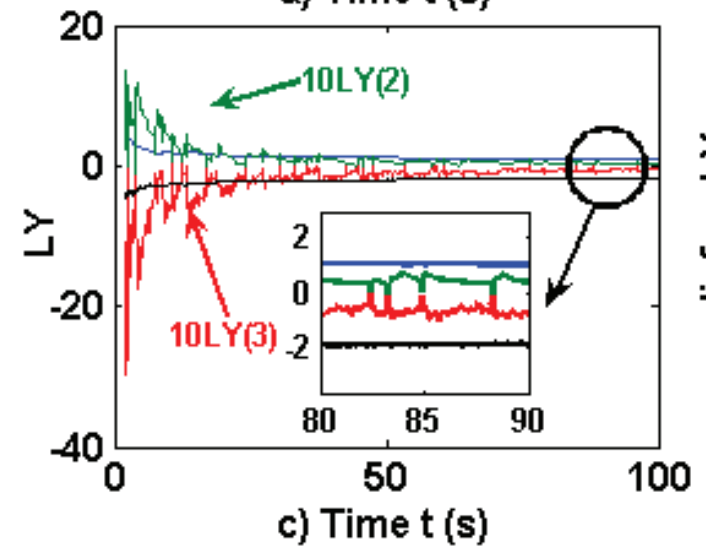

c) Time t (s)

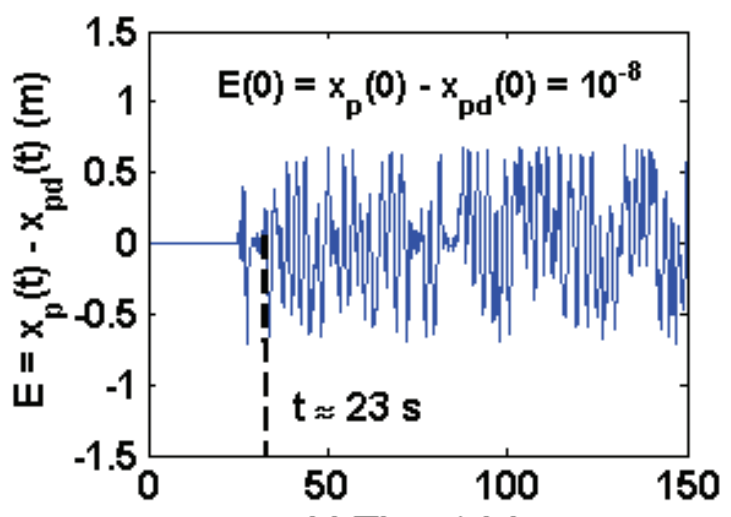

b) Time t (s)

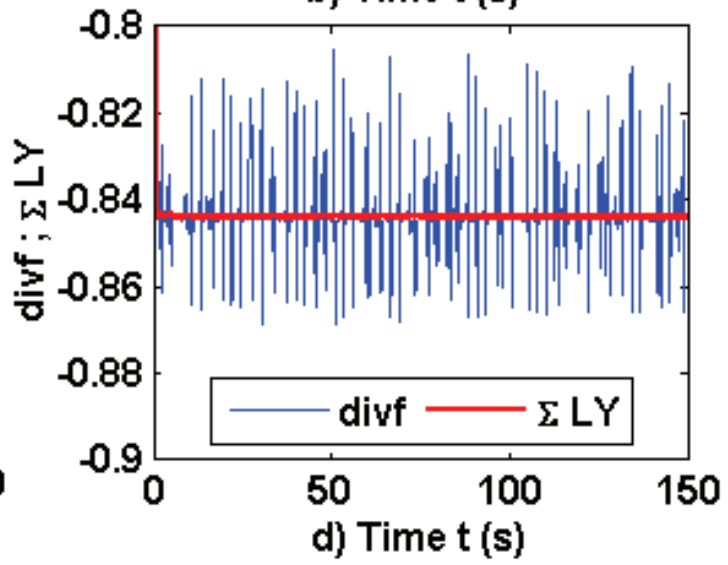

Figure 13 

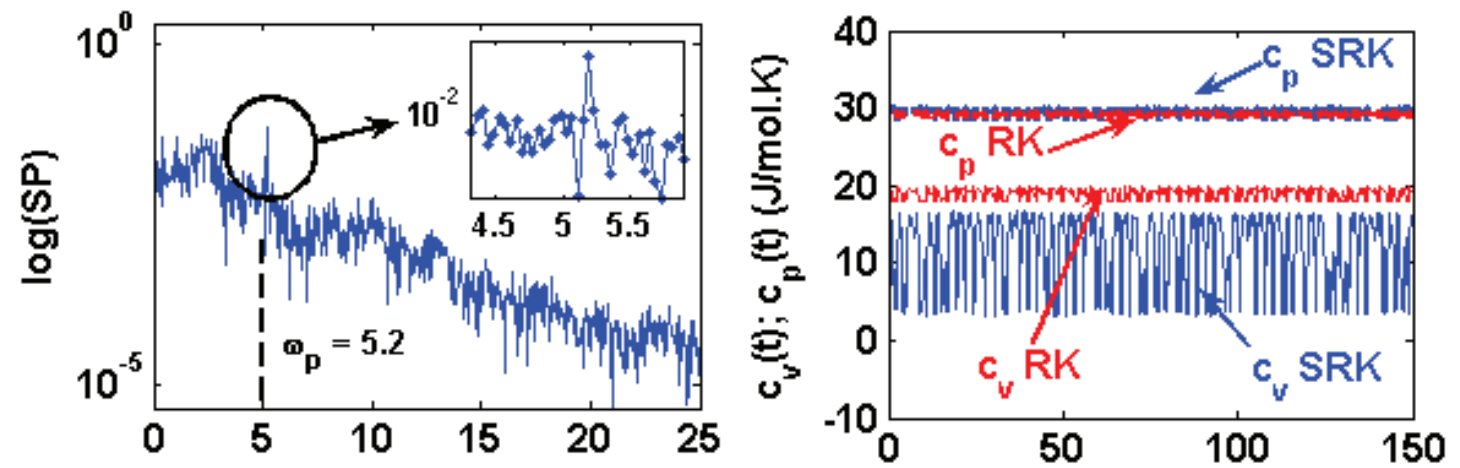

a) Frequency o (radis)
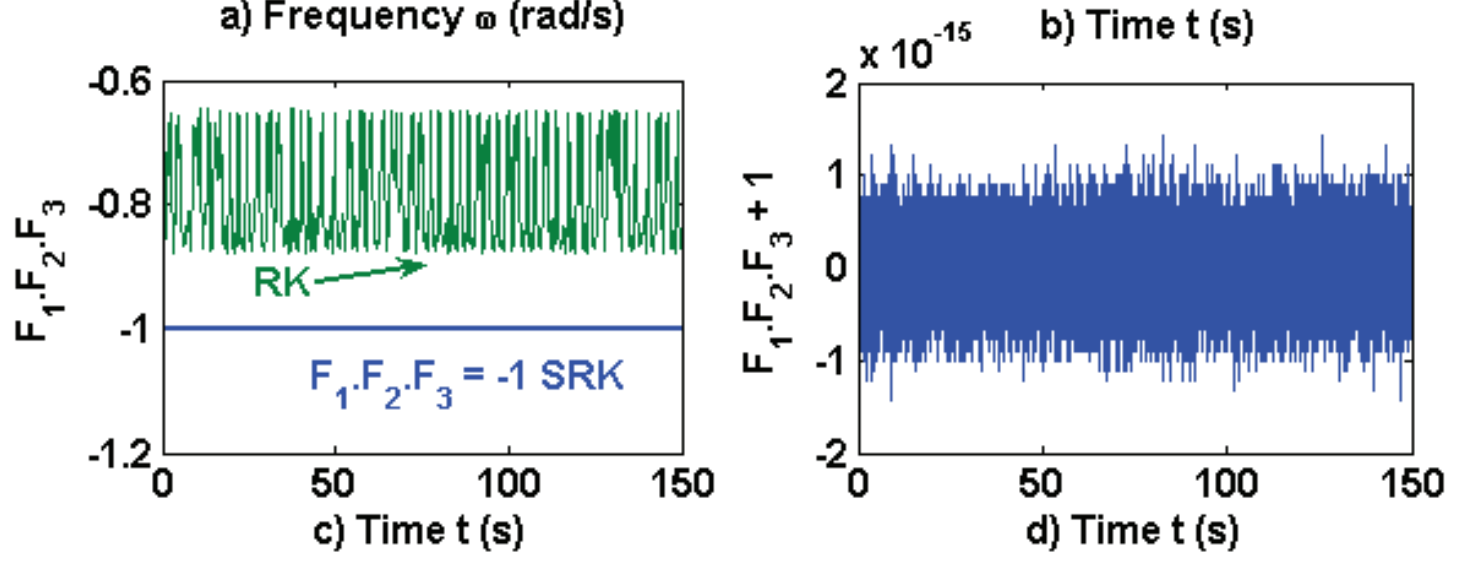

Figure 14 

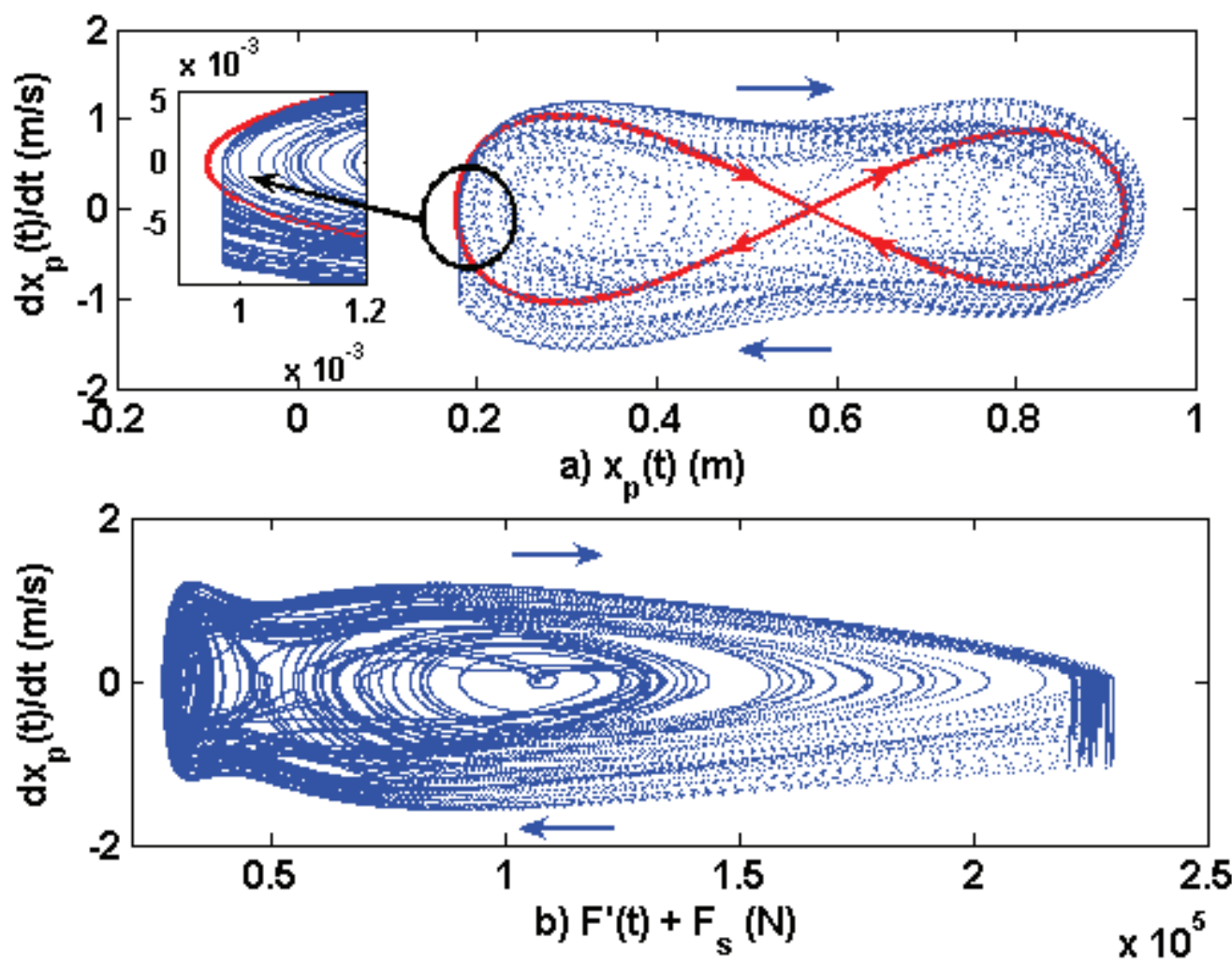

Figure 15 


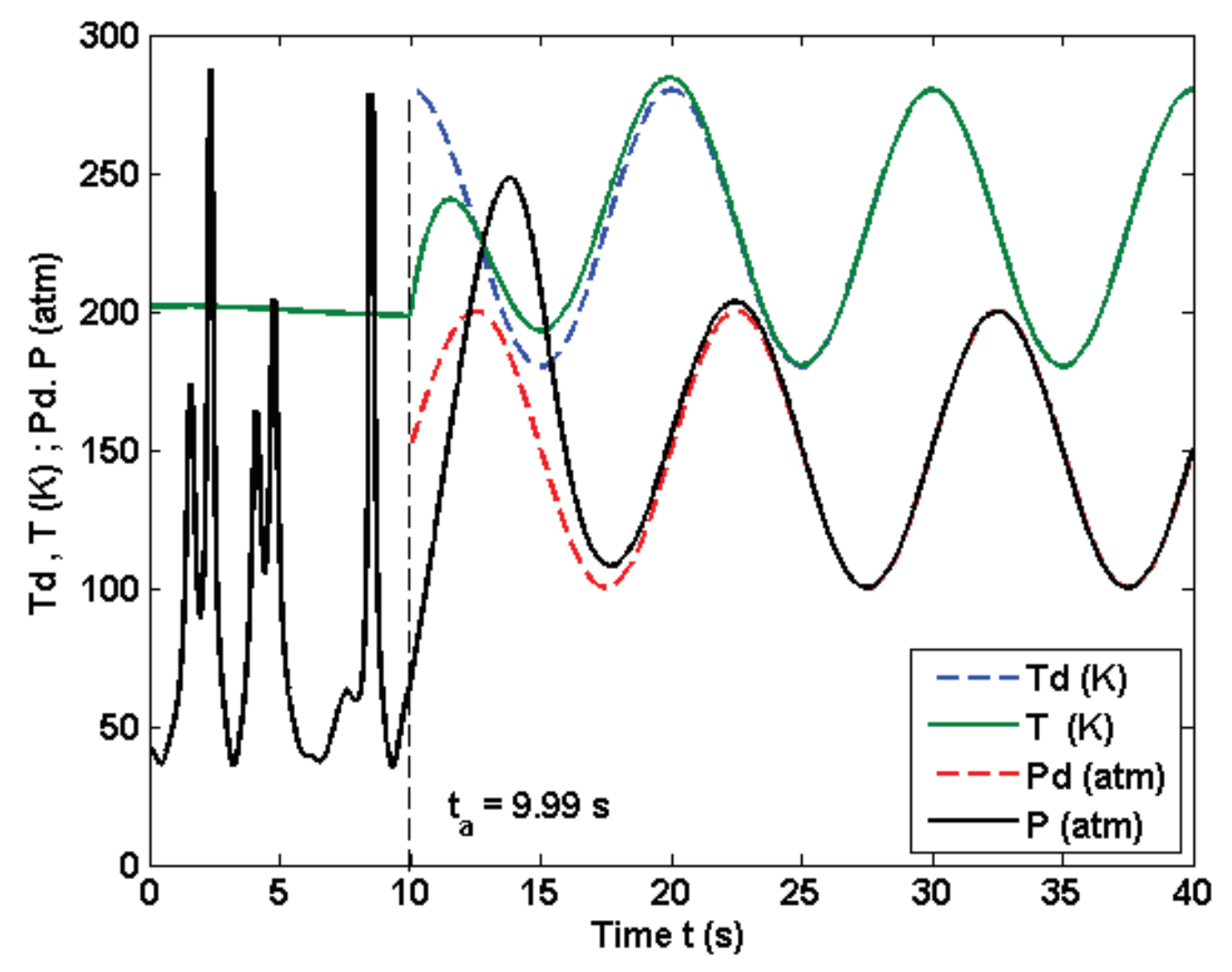

Figure 16 


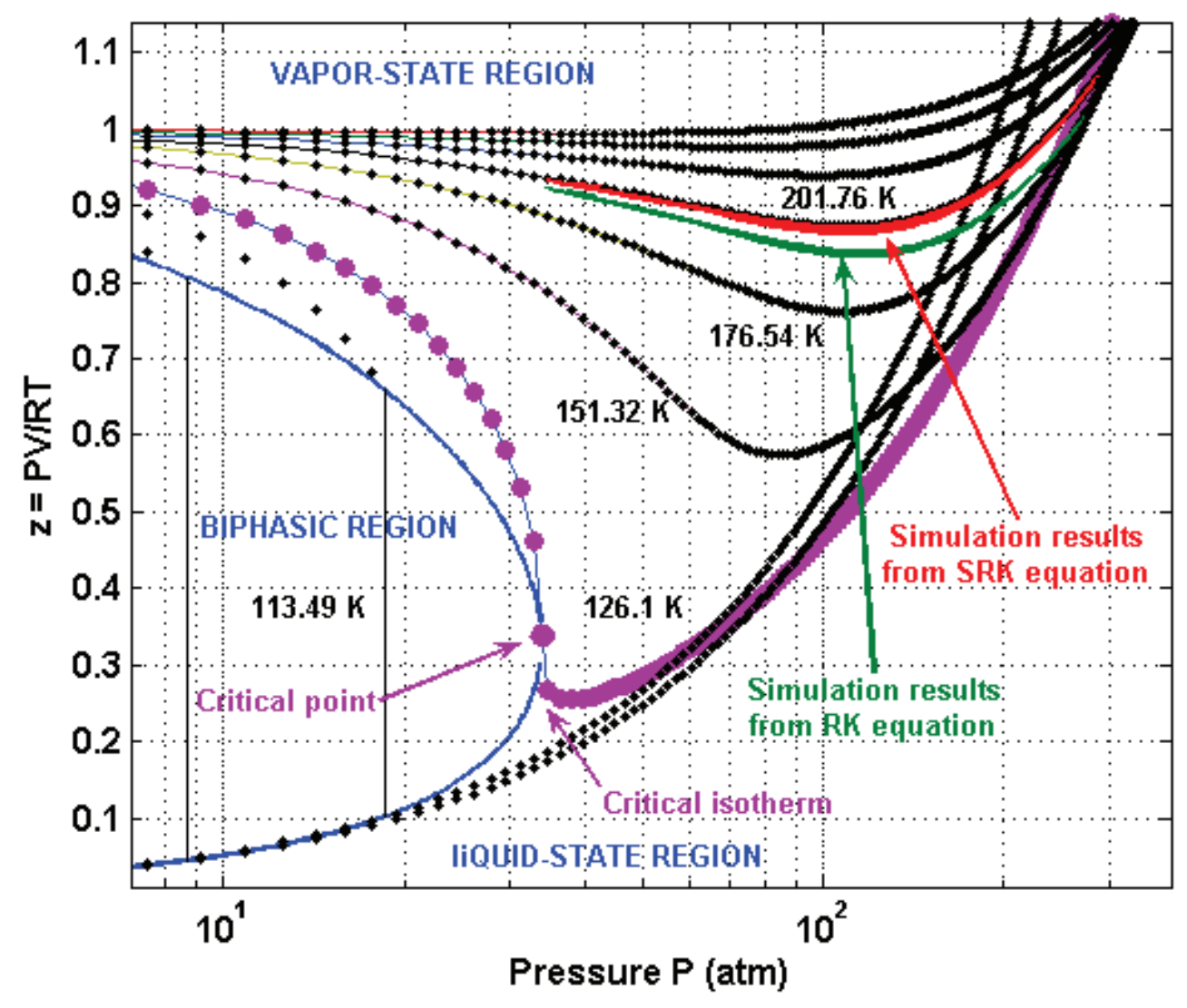

Figure 17 


\section{Legend of Figures}

Figure 1. Layout of the mobile piston inside a cylinder with nonlinear spring, damper, heating coils $\left(\mathrm{C}, \mathrm{C}_{1}\right)$, PI control loop 1, nonlinear control loop 2 and control force $\mathrm{F}^{\prime}(\mathrm{t})$. The parameter values are indicated in table 1 .

Figure 2. Reset time of the PI controller in the control loop 1 as a function of the parameters of the control loop 2. a) Taking the proportional control action $K_{p}$ as a parameter; b) Taking the coefficient $\beta$ as a parameter and c) Taking the coefficient $K_{s}$ of the heating coils in control loop 2 as a parameter.

Figure 3. a) Variation of the constants $\mathrm{K}_{1}, \mathrm{~K}_{2}$ of the nonlinear spring and the constant $\mathrm{K}$ of the control force $F^{\prime}(t)$ as a function of the steady-state pressure $\mathrm{P}_{\mathrm{s}}$, which is applied trough the control force $F^{\prime}(t)$. b) Equilibrium points of piston and equilibrium curve inside the zone of the mobile piston with three equilibrium points.

Figure 4. Equilibrium curves obtained from Eqs (25)-(27) assuming four values for $\mathrm{K}_{1}$, $\mathrm{K}_{2}, \mathrm{~K}$ and $\mathrm{P}_{1 \mathrm{~s}}$ and their corresponding equilibrium points inside the zone of the piston movement.

Figure 5. Variation of the parameters $\mathrm{p}, \mathrm{q}, \mathrm{r}$ and $\mathrm{I}_{\mathrm{h}}(\mathrm{Eq}(35))$ as a function of the piston position inside the zone where there are three equilibrium points. The shadow zone represents the values for which homoclinic orbits can appear.

Figure 6. Homoclinic orbits plotted through Eqs (43)-(45) for the values of p, q, r and $\mathrm{I}_{\mathrm{h}}$ inside the shadow zone of Fig 5. The constants $\mathrm{K}_{1}$ and $\mathrm{K}_{2}$ of the nonlinear spring and the constants $\mathrm{K}$ and $\mathrm{P}_{1 \mathrm{~s}}$ of the nonlinear control law defined by Eq (24) are indicated in Fig 5.

Figure 7. Analysis of the oscillating behavior when the equilibrium points $P_{1}$ and $P_{3}$ are weak focuses. a) Closed orbits around the equilibrium points $\mathrm{P}_{1}(0.2352)$ and $\mathrm{P}_{3}$ (0.7612) with initial piston positions of $0.3 \mathrm{~m}$ and $0.8 \mathrm{~m}$ respectively. $\mathrm{b}$ ) Position of the closed orbits with respect to the homoclinic orbit corresponding to the parameters $\mathrm{K}_{1}=$ $311.64 \mathrm{~N} / \mathrm{m}, \mathrm{K}_{2}=449.84 \mathrm{~N} / \mathrm{m}^{3}, \mathrm{~K}=680.14 \mathrm{~N} / \mathrm{m}^{2}$ and $\mathrm{P}_{1 \mathrm{~s}}=5287.7 \mathrm{~N} / \mathrm{m}^{2}$. c) Oscillations plotted as a function of the time for the simulated and approximate mechanical subsystem around the equilibrium point $\mathrm{P}_{1}$. d) Oscillations plotted as a function of the time for the simulated and approximate mechanical subsystem around the equilibrium point $\mathrm{P}_{3}$.

Figure 8. Simulation results when the whole system reaches a prescribed steady state corresponding to point $P_{3}(0.7612)$. The set point temperature is $T_{2}=200 \mathrm{~K}$, the initial cylinder pressure is $\mathrm{P}_{\mathrm{s}}=40 \mathrm{~atm}$ and the initial conditions in deviation variables are $\mathrm{x}_{0}=$ $\left[\begin{array}{llll}0.001 & 0 & 2 & 0\end{array}\right], \mathrm{K}_{\mathrm{p}}=1.827 \cdot 10^{-9}$ and $\tau_{\mathrm{i}}=0.1576 \mathrm{~s}$. The parameters of the mechanical subsystem are indicated at legend of Fig 7, whereas the parameters of the thermal subsystem are $\alpha=9.7262 \cdot 10^{-11}, \beta=1.333 \cdot 10^{-10}$ and $K_{\mathrm{s}}=7.5020 \cdot 10^{8}$. a) Steady-state piston position obtained with the SRK and RK state equations. b) Temperatures obtained from the SRK $\left(\mathrm{T}_{\mathrm{g} 1}\right)$ and $\mathrm{RK}\left(\mathrm{T}_{\mathrm{g} 2}\right)$ state equations and approximate temperature $\left(\mathrm{T}_{\mathrm{a}}\right)$ obtained from the linear system for the SRK state equation. c) Heat supply from the heating coils as a function of the time for the SRK and RK state equations. d) Pressures within the cylinder as functions of the time for the SRK and RK state equations. 
Figure 9. Simulation results corresponding to data indicated at legends of Figs 7 and 8. a) Specific heats $c_{p}$ evaluated with Eqs (52) and (55) as a function of the time for the SRK and RK state equations and specific heats $\mathrm{c}_{\mathrm{v}}$ obtained from Eq (53) as a function of the time for the SRK and RK state equations. b) Compressibility factor as a function of the time for the SRK and RK state equations. c) Product $F_{1} \cdot F_{2} \cdot F_{3}$ given by Eq (63) as a function of the time for the SRK and RK state equations. d) Values of $F_{1}, F_{2}$ and $F_{3}$ as a function of the time for the SRK and RK state equations.

Figure 10. Oscillating behavior of the whole system, for which the parameter values are indicated at legends of Figs 7 and 8. a) Piston position as a function of the time for the SRK and RK state equations. b) Temperature as a function of the time for the SRK and RK state equations. c) Heat supply from the heating coils as a function of the time for the SRK and RK state equations. d) Product $F_{1} \cdot F_{2} \cdot F_{3}$ given by Eq (63) as a function of the time for the SRK and RK state equations.

Figure 11. a) Zone of possible chaotic behaviors and curve showing the combinations of values for the frequency and amplitude of the external disturbance which do not lead to chaotic behavior. b) Melnikov's functions for several frequencies and amplitudes of the external disturbance.

Figure 12. Chaotic behavior of the whole system with a harmonic disturbance of parameters $A_{p}=0.02$ and $\omega_{p}=5.2$. Parameter values are indicated at legends of Figs 7, 8 and 9. a) Pressures within the cylinder as a function of the time for the SRK and RK state equations. b) Specific volume of the gas as a function of the time for the SRK and RK state equations. c) Temperatures of the gas as a function of the time for the SRK and RK state equations. d) Compressibility factor as a function of the time for the SRK and RK state equations.

Figure 13. Chaotic behavior of the whole system with a harmonic disturbance of parameters $A_{p}=0.02$ and $\omega_{p}=5.2$. Parameter values are indicated at legend of Figs 7, 8 and 9. a) Sensitive dependence as a function of the time for the SRK state equation. b) Evolution of the error as a function of the time with a difference of $10^{-8}$ between the initial conditions. c) Lyapunov exponents as a function of the time for the SRK state equation. d) Sum of the Lyapunov exponents and divergence of the vector field obtained from Eqs (67) and (68) assuming the SRK state equation.

Figure 14. Chaotic behavior of the whole system with a harmonic disturbance of parameters $A_{p}=0.02$ and $\omega_{p}=5.2$. Parameter values are indicated at legend of Figs 7, 8 and 9. a) Power spectral density for the piston position, which indicates the peak of the disturbance frequency. b) Specific heats $c_{p}$ evaluated with $\mathrm{Eq}(52)$ as a function of the time for the SRK and RK state equations. Specific heats $c_{v}$ obtained from Eq (53) as a function of the time for the SRK and RK state equations. c) Product $F_{1} \cdot F_{2} \cdot F_{3}$ given by Eq (63) as a function of the time for the SRK and RK state equations. d) Evolution of $\mathrm{F}_{1} \cdot \mathrm{F}_{2} \cdot \mathrm{F}_{3}+1$ as a function of the time. 
Figure 15. Chaotic behavior of the whole system with a harmonic disturbance of parameters $A_{p}=0.02$ and $\omega_{p}=5.2$. Parameter values are indicated at legend of Figs 7, 8 and 9. a) Strange attractor in the phase plane $x_{p}-d x_{p} / d t$ (portrait of the piston position versus the piston velocity) showing the overlapping of the homoclinic orbit and the constraint of the piston movement. b) Strange attractor in the phase plane $F^{\prime}(t)+F_{s}$ versus $\mathrm{dx}_{\mathrm{p}} / \mathrm{dt}$.

Figure 16. Extinction of chaotic behavior and tracking of a desired gas behavior defined by Eq (77). The parameters for the chaotic behavior are shown in the legend of Figs 14 and 15. The parameter values are given in accordance with Eqs (73) and (76) by: $t_{a}=$ $9.99 \mathrm{~s}, \omega_{\mathrm{t}}=0.6281 \mathrm{rad} / \mathrm{s}, \delta_{\mathrm{F}}=0.9, \omega_{\mathrm{nF}}=0.5111 \mathrm{rad} / \mathrm{s} ; \mathrm{K}_{\mathrm{pT}}=0.7452 \mathrm{~s}^{-1}$ and $\tau_{\mathrm{iT}}=$ $4.3478 \mathrm{~s}$.

Figure 17. Compressibility chart for the nitrogen obtained from the SRK state equation, showing the simulation results plotted in Fig 12 for the SRK state equation. The simulation results for the RK state equation are also plotted. 